\title{
REVIEW OF SUBCRITICAL SOURCE-DRIVEN NOISE ANALYSIS MEASUREMENTS
}

\author{
Timothy E. Valentine
}

Instrumentation and Controls Division

November 1999

\author{
Prepared by \\ OAK RIDGE NATIONAL LABORATORY \\ Oak Ridge, Tennessee 37831-6004 \\ managed by \\ LOCKHEED MARTIN ENERGY RESEARCH CORP. \\ for the \\ U.S. DEPARTMENT OF ENERGY \\ under contract DE-AC05-96OR22464
}


This page intentionally left blank. 


\section{CONTENTS}

Page

LIST OF FIGURES

$\mathrm{v}$

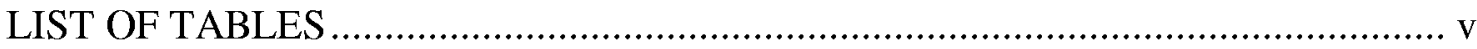

ACKNOWLEDGMENTS ......................................................................... vii

ABSTRACT …......................................................................................... ix

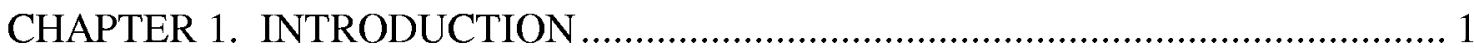

CHAPTER 2. THEORY OF SUBCRITICAL NOISE MEASUREMENT ................. 5

2.1 SIMPLE THEORY OF FISSION CHAIN FLUCTUATIONS ................. 5

2.1.1 Neutron Detection Models ................................................... 5

2.1.2 Frequency Analysis Models .................................................... 7

2.1.3 Ratio of Spectral Quantities ................................................... 9

2.1.4 Coherence Values ...................................................................... 11

2.2 Stochastic Neutron Transport..................................................... 12

2.3 Monte Carlo Simulation................................................................ 14

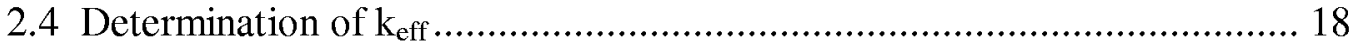

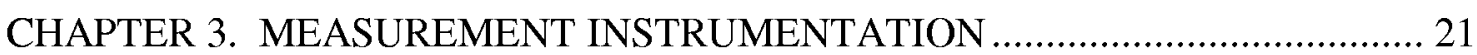

3.1 NEUTRON AND GAMMA RAY DETECTORS ............................... 21

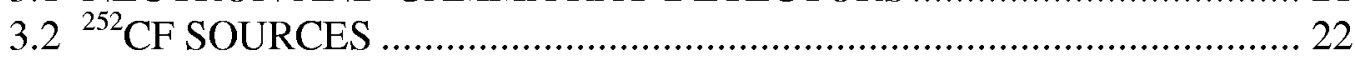

3.3 SOURCE AND DETECTION SYSTEM ELECTRONICS .................... 25

3.4 FOURIER PROCESSOR AND COMPONENTS ............................... 25

CHAPTER 4. APPLICATION OF SUBCRITICAL NOISE MEASUREMENTS .... 29

4.1 REVIEW OF SUBCRITICAL NOISE MEASUREMENTS .................. 29

4.2 SUBCRITICAL BENCHMARK MEASUREMENTS ........................ 31

4.3 IN-SITU SUBCRITICALITY MEASUREMENTS .............................. 32

4.4 PROCESS MONITORING.......................................................... 34

CHAPTER 5. EXTENSIONS OF SUBCRITICAL NOISE MEASUREMENTS....... 35

5.1 ALTERNATE NEUTRON SOURCES .......................................... 35

5.2 BI-SPECTRAL RATIO MEASUREMENT ...................................... 35

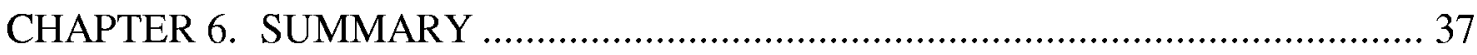

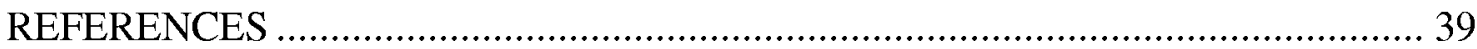

APPENDIX A. SIMPLE THEORY OF FISSION CHAIN FLUCTUATIONS ......... 45

A.1 POINT REACTOR KINETICS MODELS .................................. 45

A.2 NEUTRON DETECTION MODELS........................................... 46 
This page intentionally left blank. 


\section{LIST OF FIGURES}

Page

Figure 1 Illustration of fission chain multiplication process.................................... 2

Figure 2 Block diagram of Monte Carlo loop structure ……................................ 15

Figure $3 \quad$ Block diagram of inner loop.......................................................... 17

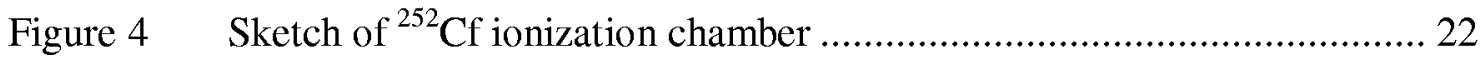

Figure 5 Source ionization chamber and high-gain fast amplifier ........................ 23

Figure $6 \quad{ }^{252} \mathrm{Cf}$ pulse-height curve as a function of the discriminator threshold...... 24

Figure 7 Typical detection system configuration ............................................. 26

Figure 8 Photograph of ZONIC WCA Fourier processor....................................... 28

Figure A.1 Fission chain for source event at time $t_{o}$ and detection event at time $t_{1} \ldots 46$

Figure A.2 Fission chains for related detector counts ............................................ 48

\section{LIST OF TABLES}

Table 1 Summary of subcritical noise measurements 
This page intentionally left blank. 


\section{ACKNOWLEDGEMENT}

The author is indebted to John T. Mihalczo for providing some of the information contained herein about subcritical source-driven noise measurements. The author would also like to thank Rafael B. Perez for providing much of the information and general understanding of the stochastic models and for the rigorous description of the perturbation relations used to interpret these measurements. Finally, the author would like to thank John K. Mattingly for the fruitful discussions concerning the many possible extensions of these measurements. John's development of the bi-spectral ratio may mitigate the few limitations of the subcritical noise measurements for in-situ applications. This work was sponsored by the Department of Energy Nuclear Criticality Safety Program under the element investigating processes for obtaining Applicable Ranges of Bounding Curves and Data (AROBCAD) related to criticality safety. 
This page intentionally left blank. 


\begin{abstract}
Subcritical source-driven noise measurements are simultaneous Rossi- $\alpha$ and randomly pulsed neutron measurements that provide measured quantities that can be related to the subcritical neutron multiplication factor. In fact, subcritical source-driven noise measurements should be performed in lieu of Rossi- $\alpha$ measurements because of the additional information that is obtained from noise measurements such as the spectral ratio and the coherence functions. The basic understanding of source-driven noise analysis measurements can be developed from a point reactor kinetics model to demonstrate how the measured quantities relate to the subcritical neutron multiplication factor. More elaborate models can also be developed using a generalized stochastic model. These measurements can be simulated using Monte Carlo codes to determine the subcritical neutron multiplication factor or to determine the sensitivity of calculations to nuclear cross section data. The interpretation of the measurement using a Monte Carlo method is based on a perturbation model for the relationship between the spectral ratio and the subcritical neutron multiplication factor.

The subcritical source-driven noise measurement has advantages over other subcritical measurement methods in that reference measurements at delayed critical are not required for interpreting the measurements. Therefore, benchmark or in-situ subcritical measurements can be performed outside a critical experiment facility. Furthermore, a certain ratio of frequency spectra has been shown to be independent of detection efficiency thereby making the measurement more robust and unaffected by drifts or changes in instrumentation during the measurement. Criteria have been defined for application of this measurement method for benchmarks and in-situ subcritical measurements. An extension of the source-driven subcritical noise measurement has also been discussed that eliminates the few technical challenges for in-situ applications.
\end{abstract}


This page intentionally left blank. 


\section{CHAPTER 1}

\section{INTRODUCTION}

The subcriticality of fissile assemblies is essential to safe processing, transportation, and storage operations. The assurance of the subcriticality of various fissile material operations is commonly obtained by criticality safety evaluations for the specific operation. The criticality safety evaluations are performed using detailed computations that have been validated using critical experiments in which the neutron multiplication factor is unity. In most cases, the actual fissile material operation does not coincide to the conditions that exist for the critical experiment, i.e. the geometry and neutron spectrum may both differ significantly between the subcritical operation and the critical experiment. These differences require that additional safety margins be included in the criticality safety evaluations ${ }^{1}$. Subcritical measurements can be used to address the use of additional safety margins by providing supplementary benchmark data for validating computation methods or by providing an in-situ measurement of the degree of subcriticality. The use of subcritical measurements would actually enhance the safety of operations with fissile materials.

Subcritical measurement methods have been under development and study for many years. These methods are all based on the same basic premise that the properties of a subcritical system can be determined by measuring the fluctuations in the fission chain multiplication process. The fluctuations in the fission chain multiplication process are dependent upon the stochastic nature of the birth and death process in the chain reaction. The birth and death process depends directly on the system geometry and composition. In a subcritical system, the fission chain process is eventually terminated because the death probability is greater than the production probability. A typical diagram of the fission multiplication process is depicted in Fig. 1. The initiating source particle denoted by $\mathrm{S}$ can be an external neutron source or an internal neutron source. The source shown in the diagram would be either an external or internal spontaneous fission source. Neutrons from the source would interact with the system resulting in fission (F), capture (C), or detection (D) of neutrons. Leakage of neutrons could be represented as a capture event because they would not produce additional neutrons nor provide to the detector response. The interaction event would depend on the geometry and composition of the system. The number of neutrons produced in fission events would fluctuate and depend on the fissile nuclide. Observe that the detection events can all be traced to the initiating source event. If the time of the source event is measurable such as in a pulsed neutron ${ }^{2}$ or randomly pulsed neutron $^{3}$ measurement, the distribution of the times between the source event and the detection event would provide a direct indication of the dynamic properties of the system. Likewise, the distribution of the times between the various detection events would also provide a direct indication of the dynamic properties of the system because these detection events are all related to the same initial source event. Such measurements are typically called Rossi- $\alpha$ measurements ${ }^{4}$ that are named after Bruno Rossi who first suggested such measurements. After many observations of the source and/or detector responses, time distribution histograms can be constructed. These histograms have an 
exponential decay that is dependent on the prompt neutron decay constant. This explanation will be further elaborated in subsequent sections of this report. The sourcejerk measurement ${ }^{5}$ is similar to the pulsed neutron measurement with the exception that the detection events are correlated with the time of the removal of the source and the subsequent decay of the fission chains is observed. Other subcritical measurements such as the inverse kinetics ${ }^{6}$ and source multiplication measurement ${ }^{7}$ rely on a physical perturbation to the system to observe the fluctuations in the fission chain process. These methods still depend on measuring events that are related to the change in the fission chain multiplication process.

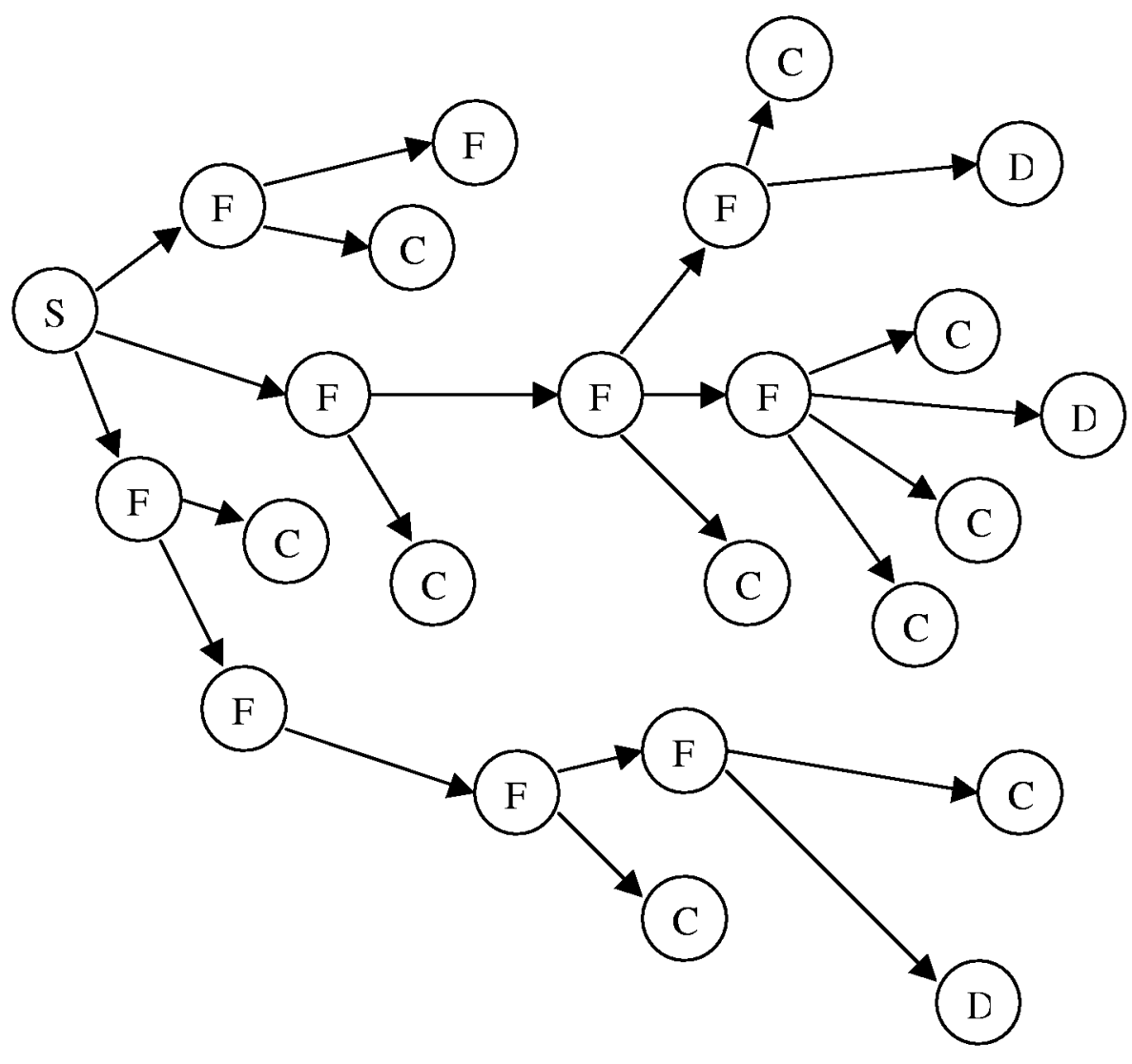

Fig. 1. Illustration of fission chain multiplication process.

The subcritical source-driven noise measurement ${ }^{8}$ (known as the ${ }^{252} \mathrm{Cf}$-source-driven noise analysis method) evolved from a simultaneous randomly pulsed neutron measurement and the Rossi- $\alpha$ measurement as an attempt to overcome some of the limitations of other methods. Other subcritical measurement methods require a calibration measurement at delayed critical or knowledge of the effective delayed neutron fraction to determine the neutron multiplication factor, $k_{\text {eff. }}$. Additionally, many of these methods required some knowledge of the detection efficiency or source intensity to 
determine $k_{\text {eff. }}$ A certain spectral ratio from the source-driven noise measurement can be directly related to the subcritical reactivity without need for a calibration measurement at delayed critical.

This report provides a description of the source-driven noise measurement and the interpretation of this method in Chapter 2. A description of frequency analysis methods is also provided in Chapter 2 to emphasize the advantages of frequency analysis. A description of the measurement method and instrumentation is provided in Chapter 3. Applications of the source-driven noise method are described in Chapter 4. A brief discussion of extensions of subcritical noise measurements is provided in Chapter 5. Finally, a summary of the report is provided in Chapter 6. 
This page intentionally left blank. 


\section{CHAPTER 2}

\section{THEORY OF SUBCRITICAL NOISE MEASUREMENT}

In this chapter the basic theory of the subcritical source-driven noise measurement is presented making the relation of this measurement method to other methods self-evident. A brief discussion of the generalized stochastic theory is presented to provide the reader with a more rigorous description of the stochastic nature of the fission chain process. A description of the Monte Carlo simulation of the source-driven noise measurement is also provided in this chapter along with a discussion of the interpretation of the measurement to determine the neutron multiplication factor.

\subsection{SIMPLE THEORY OF FISSION CHAIN FLUCTUATIONS}

\subsubsection{Neutron Detection Models}

The observation of the fluctuations in the fission chain process is accomplished by using neutron detectors. Neutron detectors may respond to events such as neutron capture, neutron fission, neutron scatter, or a combination of such events within the detector volume. Fission and scatter detectors are not necessarily terminal events. In fact, they may produce subsequent neutrons that can also contribute to the neutron fission chain. A terminal event is an absorption event in the detector without subsequent production of other neutrons. For practical purposes the following description of the detection events will only consider terminal detection events such as neutron capture. These detection models do not include the spatial or energy dependence of the detection process because the spatial dependence is treated as a point with a single energy group. The detector fieldof-view and energy dependence will be represented by the detection efficiency that is defined as detector counts per fission. Hence, the efficiency provided in these models does not represent a measurable efficiency. The following equations are based on the point reactor kinetics ${ }^{9}$ equations and are similar to those developed by Ficaro $^{10}$ and Mattingly ${ }^{11}$. Other derivations have been performed by Feynman ${ }^{12}$, Keepin ${ }^{13}$, and Uhrig ${ }^{14}$ that upon manipulation yield the same expressions. The detailed derivation of the correlation functions is provided in Appendix A.

In a source-driven noise measurement, the source event is observable in time. The source could be either an instrumented spontaneous fission source or a pulse source such as a D-T or D-D source. The time-dependent source-detector covariance function is defined as

$$
C_{s x}(t)=\frac{e_{s} e_{1} \bar{n}_{0} F_{s} e^{-a(t)}}{\bar{n} \Lambda}
$$


In this expression, $t$ is the time variable, $a$ is the prompt neutron decay constant, $F_{s}$ is the average fission rate of the source, $e_{s}$ is the efficiency for detecting the source event, $e_{I}$ is the detector efficiency in counts per fission, $\overline{v_{0}}$ is the average number of neutrons from the source, $\bar{v}$ is the average number of neutrons from fission, and $L$ is the neutron generation time. The source-detector covariance function indicates the amount of sourceinduced fission in the system.

The second part of the source-driven noise measurement is the Rossi- $\alpha$ measurement that involves the time correlation of detection events between detectors. The detector-detector covariance function including both the ${ }^{252} \mathrm{Cf}$ source and an inherent spontaneous fission source would be defined as

$$
\begin{aligned}
C_{x y}(t) & =e_{1} e_{2}\left[\overline{v(n-1)} \frac{\overline{n_{0}} F_{s}}{a \Lambda}+\overline{v_{0}\left(n_{0}-1\right)} F_{s}+\overline{v(n-1)} \frac{\overline{n_{I}} F_{I}}{a \Lambda}+\overline{v_{I}\left(n_{I}-1\right)} F_{I}\right] \\
& \cdot \frac{e^{-a|t|}}{2 a(\bar{n} \Lambda)^{2}} .
\end{aligned}
$$

In this expression, $e_{1}$ is the detector efficiency in counts per fission for the first detector, $e_{2}$ is the detector efficiency in counts per fission for the second detector, $\bar{v}_{0}$ is the average number of neutrons from the source, $\bar{v}$ is the average number of neutron from fission, $\bar{v}_{I}$ is the average number of neutrons from an inherent source, $\overline{v(v-1)}$ is the reduced second moment of the neutron emission distribution for fission, $\overline{v_{0}\left(v_{0}-1\right)}$ is the reduced second moment of the neutron emission distribution for ${ }^{252} \mathrm{Cf}$ spontaneous fission, $\overline{v_{I}\left(v_{I}-1\right)}$ is the reduced second moment of the neutron emission distribution for the inherent source, and $F_{I}$ is the spontaneous fission rate of the inherent source. The detector-detector covariance function defined by Eq. 2.7 is the same as the two-detector Rossi- $\alpha$ measurement. The detector-detector covariance function indicates the amount of source-induced and inherent source-induced fission in the system. If the measurement is performed with a single detector, the measurement is referred to as a single-detector Rossi- $\alpha$ measurement and an additional term appears in the expression for the detector auto covariance function. The single-detector auto covariance is defined as 


$$
\begin{aligned}
C_{x x}(t) & =e_{1} e_{1}\left[\overline{v(n-1)} \frac{\overline{n_{0}} F_{s}}{a \Lambda}+\overline{v_{0}\left(n_{0}-1\right)} F_{s}+\overline{v(n-1)} \frac{\overline{n_{I}} F_{I}}{a \Lambda}+\overline{v_{I}\left(n_{I}-1\right)} F_{I}\right] \\
& \bullet \frac{e^{-a|t|}}{2 a(\bar{n} \Lambda)^{2}}+e_{1} d(t)\left[\frac{\overline{n_{0}} F_{S}}{a \Lambda}+\frac{\overline{n_{I}} F_{I}}{a \Lambda}\right] .
\end{aligned}
$$

The second term appears in Eq. 2.3 because the detector signal is always correlated with itself. The single-detector auto covariance is indicative of the amount of induced fission in the system as a result of the ${ }^{252} \mathrm{Cf}$ source and the inherent source and is also indicative of the average count rate of the detectors.

The source-driven noise measurement is based on simultaneous performance of the randomly pulsed neutron and Rossi- $\alpha$ measurements. The simplistic point models for these measurements demonstrate the dependencies of these measurements on the moments of prompt neutrons from fission, the prompt neutron decay constant, and the neutron generation time. The accidental coincidences between the source and detectors and between detectors have been ignored in the previous equations to simplify the description of the physics of the measurements. Obviously the accidental coincidence rate will be a product of the count rates of the different detectors times the time interval over which the counts are acquired.

The source-driven noise measurement differs from other subcritical measurements in that the data acquisition is actually performed using Fourier processors. The reasons for the use of the Fourier processor and a brief description of the measured quantities in the frequency domain will be presented in the following section.

\subsubsection{Frequency Analysis Models}

The relationship between the covariance functions and their equivalent frequency spectra will be described in terms of the Laplace transform. Additional details concerning frequency analysis as applied to nuclear processes can be found in Ref. 14. The Laplace transform is a linear transform and is defined as ${ }^{15}$

$$
L(f(t)) \equiv F(s)=\int e^{-s t} f(t) d t .
$$

The source-detector frequency spectra can be defined as the Laplace transform of the source-detector covariance function

$$
S_{s x}(s)=L\left(C_{s x}(t)\right)=\int \frac{e_{s} e_{1} \bar{n}_{0} F_{s} e^{-a t} e^{-s t}}{\bar{n} \Lambda} d t
$$




$$
=\frac{e_{s} e \bar{n}_{0} F_{s}}{\bar{n} \Lambda} \frac{1}{(s+a)} .
$$

The detector-detector frequency spectra can be defined as the Laplace transform of the detector-detector covariance function

$$
\begin{aligned}
& S_{x y}(s)=L\left(C_{x y}(t)\right)=\frac{e_{1} e_{2}}{2 a(\bar{n} \Lambda)^{2}}\left[\overline{v(n-1)} \frac{\overline{n_{0}} F_{s}}{a \Lambda}+\overline{v_{0}\left(n_{0}-1\right)} F_{s}+\overline{v(n-1)} \frac{\overline{n_{I}} F_{I}}{a \Lambda}+\overline{v_{I}\left(n_{I}-1\right)} F_{I}\right] \\
& \int e^{-a|t|_{e}-s t} d t \\
& =\frac{e_{1} e_{2}}{2(\bar{n} \Lambda)^{2}}\left[\frac{\overline{v(n-1)}}{n_{0} F_{s}}\right. \\
& a \Lambda
\end{aligned}
$$

The Fourier transforms can be expressed in terms of the Laplace transform by setting $s=j w$. In terms of the Fourier transform, the source-detector cross spectrum is

$$
S_{s x}(w)=\frac{e_{s} e_{1} \bar{n}_{0} F_{s}}{\bar{n} \Lambda} \frac{1}{(j w+a)}
$$

The detector-detector cross spectrum is

$$
\begin{aligned}
S_{x y}(w)= & \frac{e_{1} e_{2}}{(\bar{n} \Lambda)^{2}}\left[\overline{v(n-1)} \frac{\overline{n_{0}} F_{s}}{a \Lambda}+\overline{v_{0}\left(n_{0}-1\right)} F_{s}+\overline{v(n-1)} \frac{\overline{n_{I}} F_{I}}{a \Lambda}+\overline{v_{I}\left(n_{I}-1\right)} F_{I}\right] \\
& \cdot \frac{1}{\left(a^{2}+w^{2}\right)} .
\end{aligned}
$$

The detector auto spectrum is given by

$$
\begin{aligned}
S_{x x}(w)= & \frac{e_{1} e_{1}}{(\bar{n} \Lambda)^{2}}\left[\overline{v(n-1)} \frac{\overline{n_{0}} F_{s}}{a \Lambda}+\overline{v_{0}\left(n_{0}-1\right)} F_{s}+\overline{v(n-1)} \frac{\overline{n_{I}} F_{I}}{a \Lambda}+\overline{v_{I}\left(n_{I}-1\right)} F_{I}\right] \\
& \cdot \frac{1}{\left(a^{2}+w^{2}\right)}+e_{1}\left[\frac{\overline{n_{0}} F_{s}}{a \Lambda}+\frac{\overline{n_{I}} F_{I}}{a \Lambda}\right]
\end{aligned}
$$


Source-driven noise measurements are commonly performed using Fourier processors. Fourier processing provides several advantages over time processing methods. Data acquisition in the frequency domain is performed faster than using shift registers because fewer operations are required. The computation of a frequency spectrum requires $\log _{2} \mathrm{~N}$ operations whereas the computation of a correlation function requires $N^{2}$ operations where $N$ is the number of time bins for the detector response. A more detailed discussion of the fast Fourier transform can be found in Ref. 15.

A second advantage of frequency analysis concerns removal of the detection system response function from the measured response functions. The affects of the detection system on the covariance functions were omitted in the preceding derivations of the covariance functions such that the physics of the measurements were not obscured. However, the affects of the detection electronics are important in any measurement. The measured covariance functions would actually represent the response of the system convolved with the response of the detection electronics. The actual detection of the neutrons typically occur on a very small time scale compared to the time scale of most measurements; however, the processing of the neutron event into a measurable pulse takes orders of magnitude longer. The nuclear event typically leads to atomic interactions to produce an electron current that can be measured. Therefore, the measured response, $o(t)$, is actually the convolution of the system response, $z(t)$, with the response function of the detector, $h(t)$ as defined by

$$
o(t)=\int h(u) z(t-u) d u .
$$

In the frequency domain, this convolution becomes a product of the system response with the detector electronics response.

$$
O(s)=H(s) Z(s) .
$$

By performing an electronic system calibration measurement without the fissile system being present, the affects of the detection electronics, $H(s)$, can be removed from the measured response by performing a simple division of the measured spectra divided by the calibration spectra. This calibration is only required to determine the prompt neutron decay constant from the measured frequency spectra. Otherwise this electronic system calibration is not required.

\subsubsection{Ratio of Spectral Quantities}

The most significant advantage of the source-driven noise measurement is that a certain ratio of frequency spectra can be obtained that does not depend on detection efficiency. As previously shown in the simple derivations, the detection efficiency is stated in terms of counts per fission and is not directly measurable. The spectral ratio of interest is defined as 


$$
R(w)=\frac{S_{s x}^{*}(w) S_{s y}(w)}{S_{s s}(w) S_{x y}(w)}
$$

The term $S_{s x}$ is the source-detector cross spectrum between the source $s$ and the detector $x$. The asterisk denotes taking the complex conjugate of the source-detector cross spectrum, $S_{s x}$. The term $S_{s y}$ is the source-detector cross spectrum between the source $s$ and the detector $y$. The term $S_{x y}$ is the detector-detector cross spectrum between detectors $x$ and $y$. The term $S_{s s}$ is the source auto spectrum and is simply equal to the source efficiency times the source fission rate $\left(S_{s s}=e_{s} F_{s}\right)$.

Considering the ${ }^{252} \mathrm{Cf}$ source as the only source in the system, substitution of the expressions in Eqs. 2.7 and 2.8 into Eq. 2.12 and simplifying the results yields the following expression for the spectral ratio

$$
R(w)=\frac{e_{s} \overline{n_{0}}}{\left[\frac{v(v-1)}{a \Lambda}+\frac{n_{0}\left(\frac{\left.n_{0}-1\right)}{\overline{n_{0}}}\right]}{\left[\frac{e_{s}}{|r|}+\frac{n_{0}}{\frac{n_{0}}{n_{0}}}\right]}\right.}=\frac{\overline{v-1)}}{\left[\frac{n_{0}}{|r|}\right]}
$$

As can be seen from Eq. 2.13 the spectral ratio is directly related to the reactivity of the system. The spectral ratio is also dependent on the source detection efficiency that can be measured. If the source detection efficiency is not $100 \%$, the measured spectral ratio values are divided by the source detection efficiency to remove this dependency. In most cases the source detection efficiency is greater than $99 \%$. The spectral ratio that includes an inherent source is of particular interest for interpreting measurements with plutonium. The spectral ratio including an inherent spontaneous fission source is expressed as

$$
R(w)=\frac{e_{s}^{\overline{n_{0}}}}{\left[\frac{\overline{v(v-1)}}{|r|}+\frac{\overline{n_{0}\left(n_{0}-1\right)}}{\overline{n_{0}}}+\frac{\overline{v(v-1)}}{|r|} \frac{F_{I}}{F_{s}}+\frac{\overline{n_{I}\left(n_{I}-1\right)}}{\overline{n_{I}}} \frac{F_{I}}{F_{s}}\right]} .
$$

As can be seen from Eq. 2.14, the spectral ratio that includes the affects of an inherent spontaneous fission source depends on the ratio of the inherent spontaneous fission rate to the ${ }^{252} \mathrm{Cf}$ source fission rate. These expressions are similar to those derived by others except that spatial correction factors and importance functions have been omitted in these equations. This dependence can be contained within the appropriately weighted cross sections and neutron emission probabilities. A spectral ratio that depends only on induced fission events caused by the ${ }^{252} \mathrm{Cf}$ source and is independent of the source detection efficiency has been developed that requires the measurement of higher-order correlation functions ${ }^{11}$. These higher-order correlation measurements have yet to be applied to subcriticality measurements, but they have been theoretically shown to posses higher 
sensitivity to reactivity and are independent of all inherent sources. A recommendation for the further investigation of these higher-order correlation measurements is provided later in this report.

\subsubsection{Coherence Values}

Another quantity commonly encountered in source-driven noise analysis measurements is the coherence function. The coherence function indicates the amount of correlated information between two signals. The coherence function may be defined as

$$
g_{i j}^{2}(w)=\frac{\left|S_{i j}(w)\right|^{2}}{S_{i i}(w) S_{j j}(w)}
$$

The low-frequency coherence values are used to determine the measurement time. The source-detector coherence function is independent of the source intensity but depends on the detection efficiency. This dependence on detection efficiency allows one to estimate the measurement time. The source-detector coherence function obtained from Eq. 2.15 is

$$
g_{s x}^{2}(w)=\frac{e_{s} e_{1}}{\frac{e_{1}}{\frac{n}{0}^{2}}\left[\frac{\bar{n}^{2}}{n(n-1)} \frac{\bar{n}^{2}}{a \Lambda}+\overline{n_{0}\left(n_{0}-1\right)}\right]+\frac{\bar{n}^{2} \Lambda}{a n_{0}}\left(a^{2}+w^{2}\right)} .
$$

The detector-detector coherence function may also be used to determine the measurement time. The detector-detector coherence function obtained from Eq. 2.15 is

$$
g_{x y}^{2}(w)=\frac{\frac{\left(e_{1} e_{2}\right)^{2}}{(n \Lambda)^{2}} A^{2} B^{2}}{\frac{\left(e_{1} e_{2}\right)^{2}}{(n \Lambda)^{2}} A^{2} B^{2}+\frac{\left(e_{1}+e_{2}\right) \overline{n_{0}}}{a \Lambda} A B+\frac{{\overline{n_{0}}}^{2}}{(a \Lambda)^{2}}}
$$

where

$$
A=\left[\overline{n(n-1)} \frac{\overline{n_{0}}}{a \Lambda}+\overline{n_{0}\left(n_{0}-1\right)}\right] \text {, }
$$




$$
B=\left(\frac{1}{a^{2}+w^{2}}\right)^{2}
$$

The coherence functions depend on the detector efficiency. Therefore, as the detection efficiency decreases, the fraction of correlated information between one signal and another decreases.

\subsection{Stochastic Neutron Transport}

The point reactor kinetics equation describes the time-dependent neutron behavior with a linear differential equation. This equation ignored the spatial and energy dependence of the neutron population. This dependence can be contained within the appropriately weighted cross sections and neutron emission probabilities. The time-dependent neutron behavior can be developed from a stochastic model. A stochastic process can be described as a process whose time dependence is based upon a set of transition probabilities. ${ }^{16}$

The behavior of neutrons is a stochastic process because the interactions of neutrons in a fissile system are dependent on several probability distribution functions. A stochastic process can be described with a generalized probability balance. In the case of neutron kinetics, the probability balance should account for all possible processes that may affect the neutron behavior including detection by neutron detectors. Several stochastic models have been developed for neutron noise analysis. These methods differ from one another in their point of reference. The "forward" stochastic models require that events which alter the neutron population in a time interval tfi $t+D t$ be considered in developing balance equations for the transition probability and the probability generating function. A probability balance that relates the future state of the neutron population to the present state is developed in the "forward" stochastic model. On the contrary, the "backward" stochastic model relates the current state of the neutron population to some past state. This difference is essentially a matter of point-of-view of the developer of the stochastic model. ${ }^{17}$ The basic premise of these models is that a generalized probability balance can be generated to describe the stochastic behavior of neutrons in a fissile system. A general probability balance is developed and a probability generating function is defined. The generating function is used to determine the moments of the neutron population or the detector response. The stochastic models developed by Munoz-Cobos, Perez, and $\operatorname{Verdu}^{18,19,20}$ are the most complete models that include a detailed account of the detector interaction in the neutron field. However, the basic premise will be described in terms of a limited point reactor description.

The following is a summary of the probability balance developed by Williams. ${ }^{21}$ This simple probability balance omits the neutron spatial and energy dependence and also omits delayed neutrons. A probability balance equation is developed that considers all possible events that can result in $N$ neutrons at time $t$. The following probability balance is obtained 


$$
\begin{aligned}
& P(N, t+\Delta t)=P(N-1, t) S \Delta t+P(N+1, t)(N-1) v \Sigma_{c} \Delta t \\
& +\sum_{n} p(n) P(N+1-n, t)(N+1-n) v \Sigma_{f} \Delta t+P(N, t)\left\{1-S \Delta t-N\left(\Sigma_{c}+\Sigma_{f}\right) \Delta t\right\} .
\end{aligned}
$$

The term on the left side of Eq. 2.18 is the probability that at time $t+D$ there are $N$ neutrons. The first term on the right side of Eq. 2.18 is the probability that at time $t$ there are $N-1$ neutrons which is multiplied by the probability that a source event produces one neutron within $D$. The second term is the probability that at time $t$ there are $N+1$ neutrons multiplied by the probability that neutron capture occurs within $D$. The third term is the probability that a neutron absorbed by a fissile atom produces $n$ neutrons multiplied by the probability that $N+1-n$ neutrons exist at time $t$ and the probability that in time $D t$ a fission event occurs. The final term in Eq. 2.18 accounts for the probability of no interaction. A differential equation can be derived from Eq. 2.18 by dividing by $D$ and taking the limit of Defi 0 . The resulting differential difference equation is

$$
\begin{aligned}
& \frac{d P(N, t)}{d t}=S[P(N-1, t)-P(N, t)]+v \Sigma_{c}(N+1) P(N+1, t)-v \Sigma_{t} N P(N, t) \\
& +v \Sigma_{f} \sum_{n} p(n)(N+1-n) P(N+1-n, t) .
\end{aligned}
$$

As stated by Williams, moments of this equation are obtained by defining the probability generating functions

$$
F(Z, t)=\sum_{N=0}^{\infty} Z^{N} P(N, t) \text { and } f(Z)=\sum_{n=0}^{I} Z^{n} p(n) .
$$

Multiplying Eq. 2.19 by $Z^{N}$ and summing over $N$ a partial-differential equation for the moment generating function is obtained

$$
\frac{\partial F(Z, t)}{\partial t}=(Z-1) S F(Z, t)+v\left[\Sigma_{c}(1-Z)+\Sigma_{f}(f(Z)-Z)\right] \frac{\partial F(Z, t)}{\partial Z} .
$$

The moments of the neutron population are determined by taking the derivative of $F(Z, t)$ with respect to $Z$ and evaluating at $Z=1$. The first moment is defined as

$$
\overline{N(t)}=\left.\frac{\partial F}{\partial Z}\right|_{Z=1}
$$

Evaluation of the first moment of Eq. 2.21 directly yields the point reactor kinetics equation without delayed neutrons. 


$$
\frac{d}{d \mathrm{t}} N(\mathrm{t})+\frac{r}{\Lambda} \mathrm{N}(t)=S(t)
$$

This brief derivation has shown how the probability balance along with a moment generating function can be used to determine the moments of the neutron population. In particular, it has been shown that the point reactor kinetics equation is simply the first moment of the more general probability balance. A similar probability balance can be developed to determine moments of the detector count rate. A more elaborate model would include the spatial and energy dependence in the probability balance. MunozCobos, Perez, and Verdu ${ }^{18}$ developed such a model. This model is a "backward" stochastic model and would produce an "adjoint" probability balance. In their model, the adjoint neutron transport equation is the first moment of the generalized probability balance. Using the commutation relation, the forward transport equation is obtained. In their model, they also included the detector to obtain expressions for the moments of the detection events that can be used to determine expressions for the various correlation functions.

\subsection{Monte Carlo Simulation}

The Monte Carlo method is a simulation of the stochastic model. Several books have been written that describe how Monte Carlo is used to simulate the behavior of neutrons in a fissile system. A detailed description of the Monte Carlo method can be found in Ref. 22. To perform a Monte Carlo simulation all that is required is a description of the system geometry and basic nuclear data. Given the phase-space location, energy, and direction of a source neutron the interaction probabilities are determined solely by the neutron cross sections of the material. The particle can be absorbed, scatter, cause fission, or exit through a boundary. The tracking of neutrons in a Monte Carlo simulation is the realization of the stochastic model. By sampling a large number of neutrons, the average behavior of neutrons in a fissile system can be determined. Sometimes the Monte Carlo method is formulated in terms of an integral form of the transport equation. However, this formulation is only useful for modifying the standard random walk procedure. Limiting the Monte Carlo method as a solution of a first moment equation is not necessary because any moment of the neutron population can be determined, as long the interaction process is not altered. Because typical biasing techniques are employed to reduce the variance of estimates of first moment quantities, they do not preserve the higher moments; therefore, analog Monte Carlo calculations must be performed when analyzing quantities that are directly related to the higher moments of the neutron populations. Because the use of average quantities reduces the statistical fluctuation of the neutron population, average quantities such as the average number of neutrons from fission are not used; instead, appropriate probability distribution functions are sampled.

The Monte Carlo codes used for this type of analysis were developed to simulate sourcedriven noise analysis measurements. The source and detector responses are accumulated and the resulting sequences segmented into data blocks. A data block is a sample of the detector response for a specified period of time. The auto and cross spectra are 
accumulated and averaged over many blocks of data. There are two codes available to simulate the frequency analysis measurement. The first is the Monte Carlo code KENO-NR ${ }^{23}$ that is a modified version of KENO- $\mathrm{Va}^{24}$ and uses group averaged neutron cross sections. This code calculates the auto- and cross spectra between a source and neutron detectors. The other code is MCNP-DSP ${ }^{25,26}$ that is a modified version of MCNP4a ${ }^{\text {TM, } 27}$. MCNP-DSP is a continuous energy Monte Carlo code which calculates the auto- and cross spectra and auto-and cross correlation functions between a source and detectors for both neutrons and gamma rays. The Monte Carlo calculation does not impose limitations on the spatial dependence of the simulation except for the accuracy of representing physical systems. The only limitation of the energy dependence is that imposed by the cross section data files whether continuous or group averaged and that imposed by the representation of the energy of neutrons and/or gamma rays from fission.

The Monte Carlo calculation typically proceeds by tracking a specified number of source particles and their progeny. However, in the KENO-NR and MCNP-DSP Monte Carlo calculations the tracking procedure consists of an additional outer loop over data blocks because the time and frequency statistics are averaged over many data blocks. The outer loop sets the current data block and the inner loop tracks events following source fission in the current data block. This is illustrated schematically in Fig. 2. The outer loop starts with $n p s=1$. If $n p s$ is greater than the number of specified blocks to be accumulated $(b k s)$, then the final outputs are obtained. Otherwise, the number of source events per data block $(n s d p b)$ is sampled from a Poisson distribution. The source particles and their progeny are then tracked until the particles are either absorbed or escape from the system. After all source particles and progeny for a given data block have been tracked, the block counter is incremented and the process repeats until all blocks have been accumulated.

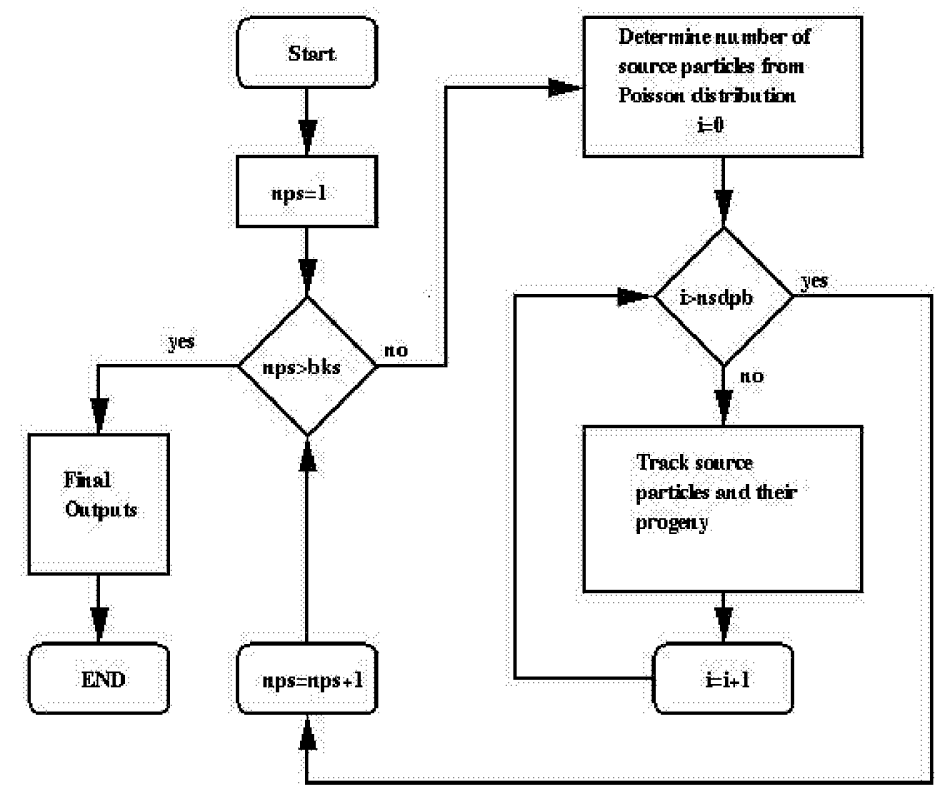

Fig. 2. Block diagram of Monte Carlo loop structure.

\footnotetext{
${ }^{\mathrm{TM}} \mathrm{MCNP}$ is a trademark of the Regents of the University of California, Los Alamos National Laboratory.
} 
A block diagram of the inner loop structure is given in Fig. 3. The inner loop begins by obtaining information about the source event. The source is treated as a point source whose directional distribution is either isotropic or determined from an appropriate distribution function. The times of the source fission events are uniformly distributed within the data block. The energy of the ${ }^{252} \mathrm{Cf}$ neutrons is sampled from a corrected Maxwellian distribution. The number of neutrons from the spontaneous fission of ${ }^{252} \mathrm{Cf}$ is sampled from a measured distribution. This information is stored in the bank for all but one of the neutrons for the given source spontaneous fission. Next, the distance to the cell boundary is determined from the geometry description of the region and the direction of the source neutron, and the distance to collision is determined probabilistically from the total macroscopic cross-section of the material in which the source is located. If the distance to collision is shorter than the distance to the cell boundary, then the collision type and time are determined from the direction and velocity of the neutron; otherwise, the particle is transported to the boundary of the region and the process is repeated. The type of collision is probabilistically determined from the nuclear cross-section data. For example, the probability of fission event is determined from the ratio of the fission crosssection to the total cross-section. If a fission event occurs, the number of neutrons from fission are sampled from the appropriate probability distributions, the fission neutron directions are sampled isotropically, and their energies are sampled from the fission spectrum of the target nucleus. The birth time of the secondary particles is the sum of the source particle birth time and the transit time to the collision site. These progeny would then be stored in the bank to be tracked latter. If the collision event occurred in the detector material, the detector response at the time of collision is incremented. If the particle survives the collision, the distance to the cell boundary is recalculated along with the distance to collision and the process is repeated. If the particle is absorbed or causes fission, then the next particle from the bank is retrieved and the distance to the boundary and the distance to collision are determined for this particle. If there are no particles in the bank, the inner loop counter is incremented. All particles are tracked until they are either absorbed or escape from the system. After all source and secondary particles for a given data block have been tracked, the detector responses are accumulated. This procedure is repeated for the specified number of data blocks to obtain average estimates of the detector responses. The auto spectra and cross spectra are estimated from the detector responses by complex multiplication of the Fourier transform of the data blocks and averaged over blocks.

In these Monte Carlo calculations, the detector material, type, and any energy thresholds are specified. The detector response is segmented into time bins for each data block whose time width is specified. There are three types of detectors available in these calculations: capture, scatter, and fission detectors. The detector response of capture detectors is due to neutron absorption followed by emission of secondary charged particles that ionize the detection media and produce an electrical pulse proportional to the kinetic energy of the secondary charged particle. In the Monte Carlo simulation, neutron absorption in the detector material results in a count at the appropriate time in the data block. In KENO-NR, all neutron absorptions in the detector media result in a count. In MCNP-DSP, a specified fraction of the neutron absorptions in the detector media lead to a count to allow for detector thresholds typically set in the measurements. Scattering 
detectors are those in which the detector response is due to neutron scattering in the detection media. These detectors are typically used to simulate liquid and/or plastic scintillators. To observe a count in a scattering detector, the neutron must deposit enough energy to the recoil nucleus to excite electrons in the scintillation material which produce light that is converted into an electrical pulse by a photomultiplier tube. In KENO-NR, the response of the scattering detectors is determined by the energy of the incident neutron. In MCNP-DSP, the response of the scattering detector is determined by the neutron energy deposition in the detection media and multiple scattering events of particles in the detectors are taken into account. The MCNP-DSP treatment is more realistic than the KENO-NR treatment of scattering detectors. In fission detectors, the fission fragments travel through the detection media ionizing the atoms in the detector. The large energy release per fission allows for easy discrimination of other events that may also produce ionized atoms in the detector. In the calculations, a count is registered each time a fission event occurs in the detection media, and the fission neutrons are stored for tracking. In MCNP-DSP, gamma rays can also contribute to the detector response for capture and scatter detectors if the calculation is a coupled neutron-photon calculation.

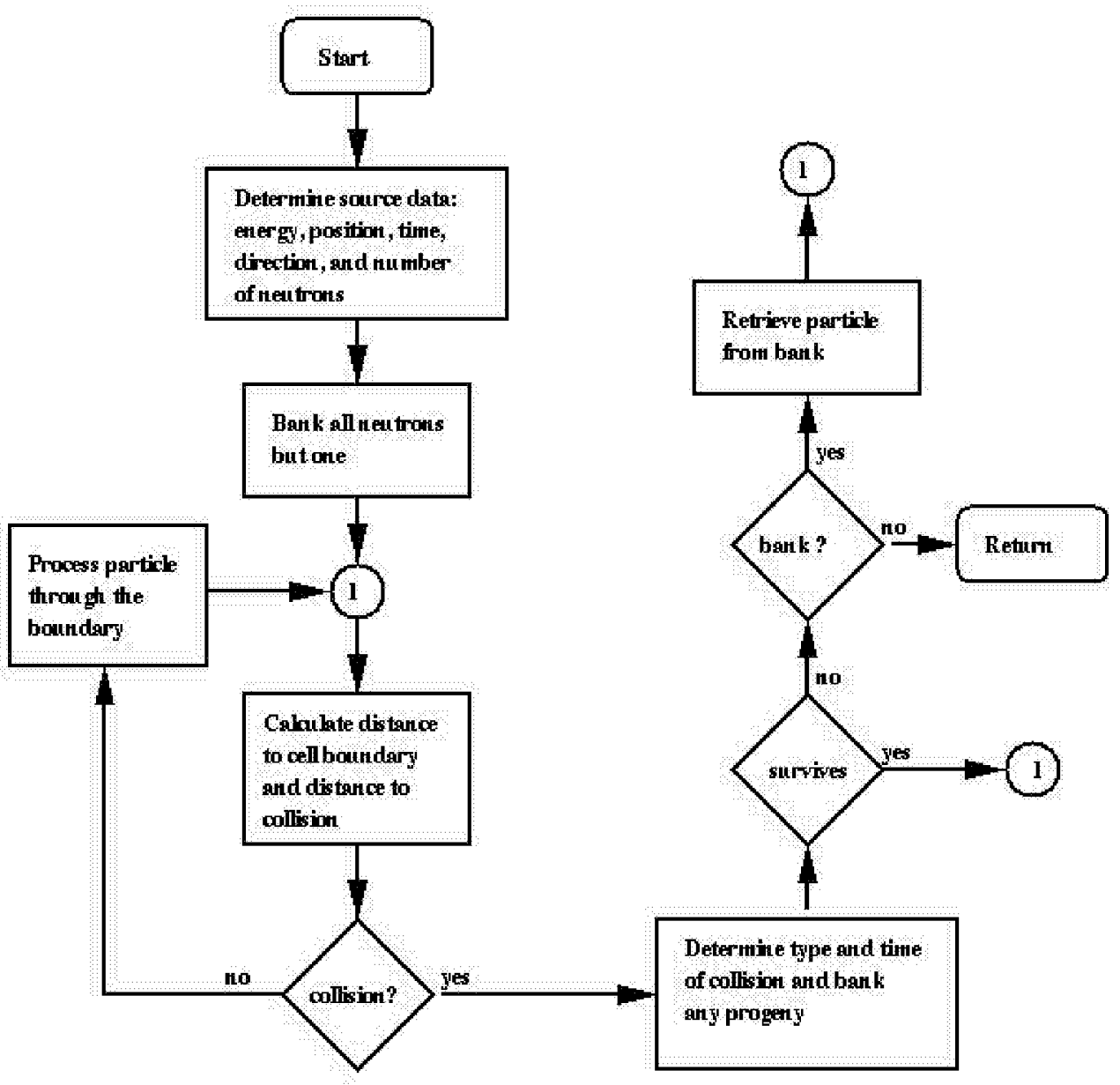

Fig. 3. Block diagram of inner loop. 


\subsection{Determination of $\mathbf{k}_{\mathrm{eff}}$}

Subcritical measurements were developed to study the dynamic behavior of neutrons in a fissile system. One common aspect of this was to determine the neutron multiplication factor. Interpretation of measurements requires some model to relate what is measured to $k_{\text {eff. }}$. In the past, the subcritical noise measurements were interpreted using equations developed from point kinetics models for the time-dependent behavior of neutrons in the subcritical configuration. This limited the application of the measurement to situations in which point kinetics was applicable. The most general model to relate the measured quantities to $k_{\text {eff }}$ would involve the use of the generalized stochastic model developed by Munoz-Cobos et al. Although an analytical solution of subcritical noise measurements in terms of the stochastic model is not practical, the Monte Carlo method as previously described provides a means to simulate the subcritical measurements and to also calculate $k_{\text {eff. }}$ In fact, the same Monte Carlo code and nuclear data can be used for simulation of subcritical measurements and for $k_{\text {eff }}$ calculation.

The Monte Carlo codes are used to interpret the measurement by performing a calculation of the measured parameters and a separate eigenvalue calculation. For example, a comparison of measured and calculated values of the spectral ratio can be used to obtain the "experimental" $k_{\text {eff. }}$. If the measured and calculated values of the spectral ratio are in agreement, then the bias in the spectral ratio is zero. The bias in the spectral ratio is defined as the difference between measured and calculated values of the spectral ratio $\left(R_{m}-R_{c}\right)$ where $R_{m}$ is the measured value and $R_{c}$ is the calculated value. First order perturbation theory can be used to obtain an expression that can be used to determine the "experimental" $k_{\text {eff }}$ and the bias in the $k_{\text {eff. }}$. The low-frequency value of the spectral ratio has been shown in numerous experiments to be linear with $k_{\text {eff }}$ over a wide range of values of $k_{\text {eff }}$ with the values of $k_{\text {eff }}$ being interpreted using point kinetics models. Given the linear dependence of the spectral ratio with $k_{\text {eff }}$, the bias in the spectral ratio varies linearly as the bias in $k_{\text {eff }}\left(k_{m}-k_{c}\right)$. To determine the "experimental" $k_{\text {eff }}$ value and its bias, the Monte Carlo models are slightly perturbed and new values of the spectral ratio $\left(R_{p}\right)$ and $k_{\text {eff }}\left(k_{p}\right)$ are obtained. If the linear dependence is valid, then the perturbation calculations can be used to obtain the "experimental" $k_{\text {eff, }}, k_{m}$, and its bias using the following linear relationship

$$
\frac{R_{m}-R_{c}}{k_{m}-k_{c}}=\frac{R_{p}-R_{c}}{k_{p}-k_{c}} .
$$

This methodology simply uses a linear interpolation or extrapolation between the standard and perturbed values of the spectral ratio and $k_{\text {eff }}$ to determine the "experimental" $k_{\text {eff. }}$ Using this relationship, the value of $k_{m}$ can be determined along with its bias $k_{m}-k_{c}$. Propagation of error is used to obtain the uncertainty in $k_{m}$ and its bias. Even if the measured spectral ratio value and the calculated value agree, the perturbation analysis is performed. This is required to equate the uncertainty in the measured spectral ratio to the uncertainty in the inferred $k_{e f f}$ value. 
Perez et. al. ${ }^{28}$ have demonstrated that the relationship given in Eq 2.24 was valid using first order perturbations of the Greens functions expressions for $k_{\text {eff }}$ and the spectral ratio. In the paper, Perez summarized the relationships previously derived for $k_{\text {eff }}$ and the spectral ratio in terms of a Greens function of the transport equation. By performing perturbations of the Greens function, relationships between the standard values of the spectral ratio or $k_{\text {eff }}$ could be related to the perturbed values. Perturbing the Greens function yields the following relationship between the standard and perturbed spectral ratio values

$$
R_{p}-R_{c}=A_{s}\left(a_{p}-1\right)
$$

In this expression $A_{s}$ contains the dependency on the Greens function and $a_{p}$ is the ratio of the perturbed parameter to the unperturbed parameter. A similar relationship is obtained for the perturbed and standard $k_{e f f}$ values

$$
k_{p}-k_{c}=Z_{s}\left(a_{p}-1\right)
$$

In this expression $Z_{s}$ contains the dependency on the Greens function. Note that the terms $A_{s}$ and $Z_{s}$ are different but both depend on the Greens function of the transport equation. The bias in the spectral ratio calculation was defined as $R_{m}-R_{c}$ and can be equated to the uncertainty of the Greens function parameters

$$
R_{m}-R_{c}=A_{s}\left(a_{m}-1\right)
$$

This essentially implies that the calculated spectral ratio differs from the measured spectral ratio because the nuclear data and the Monte Carlo model do not sufficiently represent the measurement conditions. Likewise, the difference between the measured $k_{m}$ and the calculated $k_{c}$ could be equated to the same uncertainty of the Greens function parameters

$$
k_{m}-k_{c}=Z_{s}\left(a_{m}-1\right)
$$

The ratio of Eq. 2.27 to Eq. 2.25 and the ratio of Eq. 2.28 to Eq. 2.26 are equal

$$
\frac{R_{m}-R_{c}}{R_{p}-R_{c}}=\frac{\left(a_{m}-1\right)}{\left(a_{p}-1\right)}=\frac{k_{m}-k_{c}}{k_{p}-k_{c}} .
$$

The results of the perturbation analyses directly yielded Eq 2.24.

Ideally, the "experimental" $k_{\text {eff }}$ value should not depend on the method of perturbation. However, the bias in $k_{m}$ would depend on the perturbation. This methodology had been 
applied to successfully interpret subcritical measurements with high-enriched uranium metal castings in storage and with uranyl nitrate solutions to demonstrate the independence of $k_{m}$ on the perturbation. ${ }^{29,30}$ In the actual applications, multiple perturbation calculations are performed. The experimental $k_{m}$ is thus defined as the average of the "experimental" $k_{\text {eff }}$ values

$$
k_{m}=\frac{1}{N} \sum_{i=1}^{N} k_{m}^{i}
$$

where $k_{m}^{i}$ is the experimental $k_{m}$ for the $i$ th perturbation. The resulting standard deviation of the experimental $k_{m}$ is thus

$$
s_{k m}^{2}=\frac{1}{N} \sum_{i=1}^{N} s_{k}^{2}
$$

In the limit of known uncertainty in the calculated quantities, the uncertainty in the experimental $k_{\text {eff }}$ would be simply the uncertainty in the measured spectral ratio value times the average square of the sensitivity coefficient

$$
s_{k m}^{2}=s_{R m}^{2} \frac{1}{N} \sum_{i=1}^{N}\left[\frac{k_{p}^{i}-k_{c}}{R_{p}^{i}-R_{c}}\right]^{2}
$$

The expression in Eq. 2.32 demonstrates as previously mentioned that even if the measured and calculated spectral ratio values agree a perturbation analysis must be performed to determine the actual uncertainty in the experimental $k_{m}$. 


\section{CHAPTER 3}

\section{MEASUREMENT INSTRUMENTATION}

This section provides a description of the instrumentation that is required to perform a subcritical noise measurement. A brief discussion of detectors is provided along with a discussion of the ${ }^{252} \mathrm{Cf}$ sources. A description of Fourier processors and subsequent components is also provided.

\subsection{NEUTRON AND GAMMA RAY DETECTORS}

This section provides a review of detectors used in subcritical noise measurements. References 29 and 30 provide detailed descriptions of the operating principles of neutron and gamma ray detectors. A variety of detectors have been used in subcritical noise measurements. Measurements have been performed with the detectors operating in both the pulse mode and current mode. The types of detectors that have been used include ${ }^{3} \mathrm{He}$ proportional chambers, fission counters, $\mathrm{BF}_{3}$ and boron-lined counters, Li-glass scintillators, NaI scintillators, plastic scintillators, liquid scintillators, and composite Liglass plastic scintillators. Selection of the detector type depends on the particular application. The detection efficiency is the primary consideration and must be sufficiently high such that measurements can be performed in a short time. Secondly, the response time of the detector must be shorter than the response time of the system being measured. Ionization chambers and proportional counters have relatively slow time responses as compared to scintillation detectors. For fast metal systems, scintillation detectors are more practical because their time response is on the order of nanoseconds and is shorter than the time response of many fast metal systems. On the other hand, ionization chambers and proportional counters are best suited for thermal systems. The response time of ionization chambers and proportional counters is on the order of microseconds and is shorter than the time response of typical thermal systems. The final point to consider is the environment in which the detector will be placed. The temperature and radiation environments are the two most important factors to consider. Detectors whose response changes drastically as the temperature changes should not be used if the temperature environment is changing. The detection efficiency of some scintillation detectors decreases as the temperature increases. Likewise, care should be taken when selecting detectors to operate in high radiation fields. In high gamma radiation fields more than $10^{3} \mathrm{Rad}$, fission chambers are the most practical detectors to use because they are insensitive to gamma radiation. High-temperature, high-sensitivity fission counters have been developed at ORNL for specific measurements with spent nuclear fuel. ${ }^{31}$ However, fission chambers have relatively low detection efficiency. For example, a 1-inOD, 20-in-long fission counter with 1 gram ${ }^{235} \mathrm{U}$ has a thermal neutron sensitivity of 0.88 counts per second per neutron/ $\left(\mathrm{cm}^{2} \mathrm{~s}\right)$. A 1-in-OD, 20-in-long ${ }^{3} \mathrm{He}$ proportional counter at 4-atm pressure has a thermal neutron sensitivity of 53 counts per second per neutron $/\left(\mathrm{cm}^{2}\right.$ s). The ${ }^{3} \mathrm{He}$ proportional counter is a factor of 60 more sensitive to thermal neutrons. 


\section{$3.2 \quad{ }^{252}$ Cf SOURCES}

The most common source used in subcritical noise measurements is the ${ }^{252} \mathrm{Cf}$ spontaneous fission source. However, other neutron sources such as a D-T or D-D neutron generator could be used. ${ }^{32}$ The ${ }^{252} \mathrm{Cf}$ source is contained in an ionization chamber that produces pulses due to the alpha decay and the spontaneous fission of ${ }^{252} \mathrm{Cf}$. The alpha decay rate is approximately 32 times the spontaneous fission rate. The designs of these chambers are parallel plate, annular, and spherical with the most common design being the parallel plate chamber. A sketch of a parallel plate ionization chamber is shown in Fig. 4.

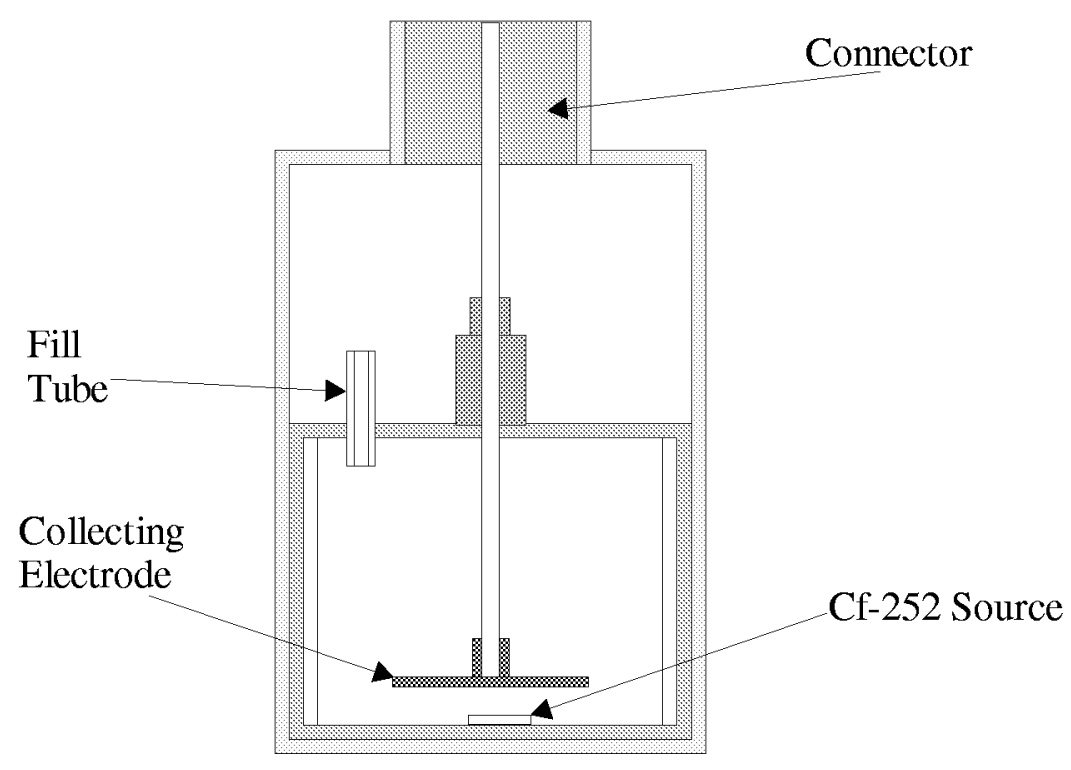

Fig. 4. Sketch of ${ }^{252} \mathrm{Cf}$ ionization chamber.

Pulses from the source ionization chamber are typically supplied to a high-gain fast amplifier for pulse processing. The amplifier produces a nuclear instrument module (NIM) pulse for each alpha decay or spontaneous fission. A photograph of the source ionization chamber connected to a high-gain fast amplifier is shown in Fig. 5. The highgain fast amplifier output is sent to a constant fraction discriminator. The fraction of spontaneous fission events is determined from a pulse height curve of the ${ }^{252} \mathrm{Cf}$-source count rate as a function of the discriminator threshold setting. The discriminator only produces an output pulse if the input pulse exceeds the specified voltage amplitude. 


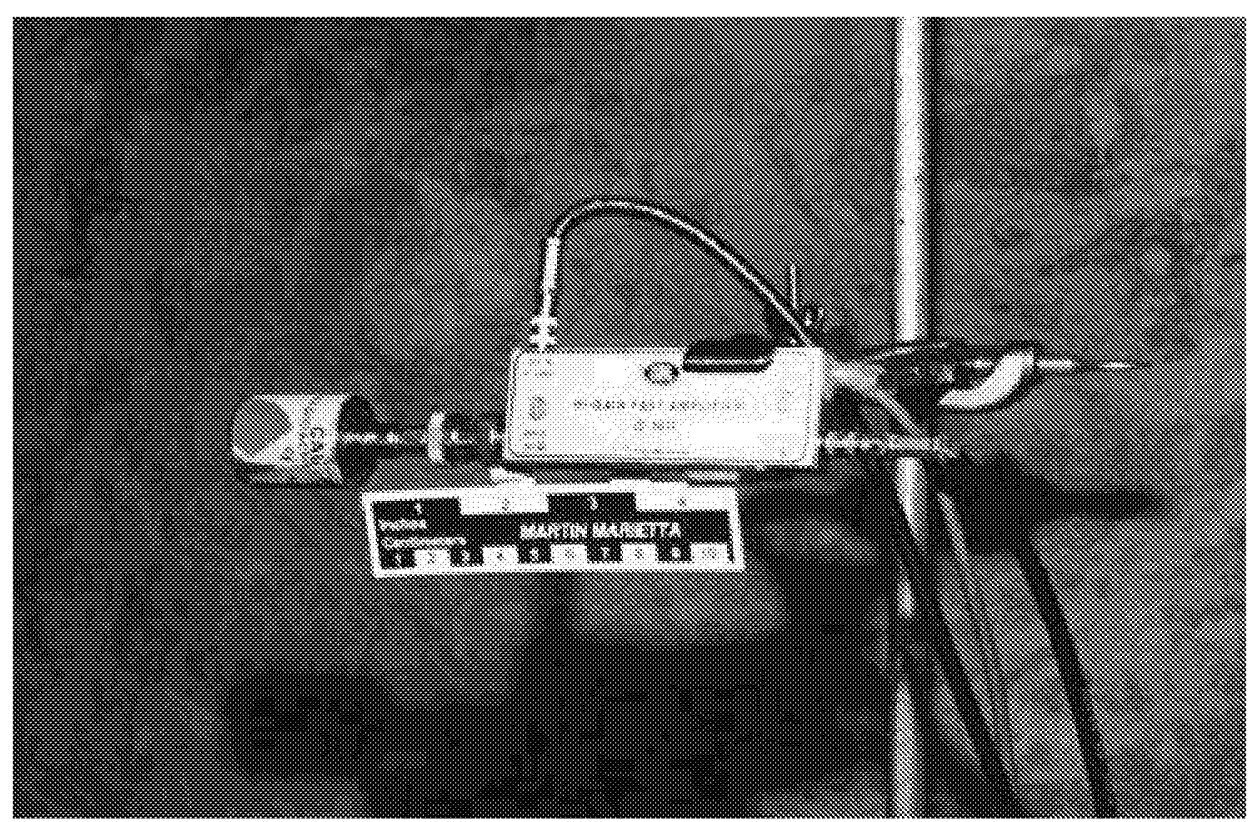

Fig. 5. Source ionization chamber and high-gain fast amplifier.

A typical plot of the count rate as a function of the discriminator threshold voltage is shown in Fig. 6. The ionization chamber pulses produced by the alpha particles are much lower in amplitude than those produced by the fission fragments. The heavier fission fragments lose more energy in the chamber gas. The alpha decay of the ${ }^{252} \mathrm{Cf}$ source is observed for low threshold settings as noted in the $35-\mathrm{mV}$ region of the curve. As the threshold is increased, the alpha particle events in the chamber are discriminated against and only pulses from the fission fragments are observed over the relatively flat portion of the pulse height curve. Further increase of the threshold eliminates some of the fission fragment pulses and causes the count rate to decrease. The discriminator threshold is set near the center of this curve so that approximately $99 \%$ of the spontaneous fission events are counted.

The source ionization chamber is typically operated in the pulse mode although measurements have been performed operating the source ionization chamber in the current mode. Current mode operation requires reference measurements be performed with a smaller source that can be operated in either the pulse or current mode to determine the contribution of alpha particles to the source current. Although the pulse height distribution curve can be used to determine the region where predominantly fission fragments are counted, reference measurements with a standard source are typically performed to determine the fraction of fission events that are counted. In most cases, more than $99 \%$ of the fission fragments are counted. 


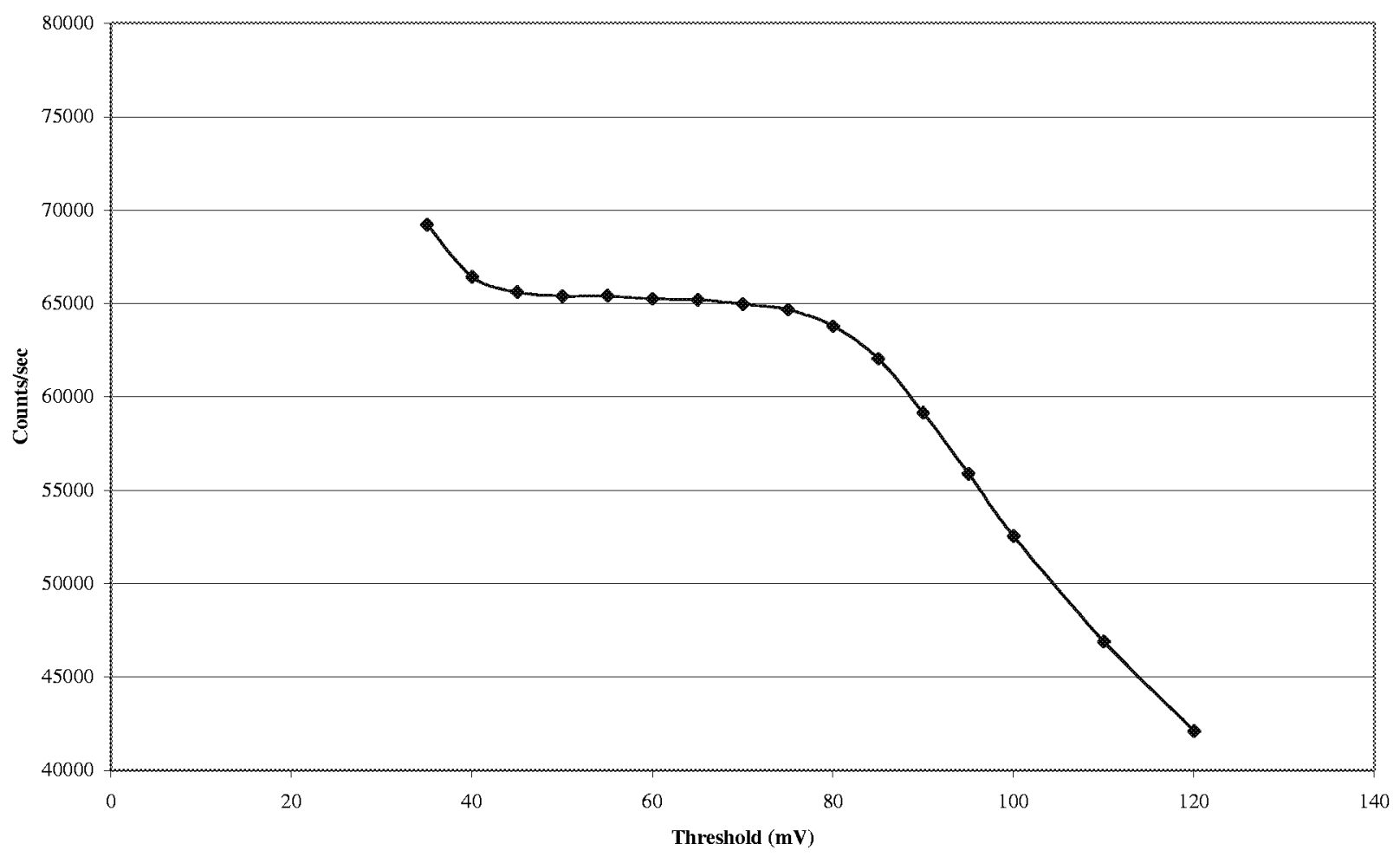

Fig. 6. ${ }^{252} \mathrm{Cf}$ pulse-height curve as a function of the discriminator threshold.

Sources with $1 \mu \mathrm{g}$ of ${ }^{252} \mathrm{Cf}$ have been routinely manufactured at the Isotopes Production Facility at Oak Ridge National Laboratory and have also been manufactured at several Russian Institutes. These sources have a typical pulse height distribution as shown in Fig. 6. Larger ${ }^{252} \mathrm{Cf}$ sources have been manufactured with up to $15 \mu \mathrm{g}$ of ${ }^{252} \mathrm{Cf}$. However, sources greater than $5 \mu \mathrm{g}$ of ${ }^{252} \mathrm{Cf}$ would require the chamber to be operated in the current mode because of limitations of electronics components and add an additional complication to performing the measurement. Recall that the spectral ratio is dependent on the source efficiency. One way to circumvent the use of the current mode is to use multiple source ionization chambers. Each individual chamber could be operated in the pulse mode and the signals could be summed together prior to being sent to the Fourier processor. 


\subsection{SOURCE AND DETECTION SYSTEM ELECTRONICS}

The electronic system consists of components that are used to convert the detector signals to an analog signal that can be digitized by the Fourier analyzer. As previously stated, the ${ }^{252} \mathrm{Cf}$ source is connected to a high-gain fast amplifier (AMP) with a high voltage (HV) input for the ionization chamber and a low voltage (DC) input to power the amplifier. The output is sent to a constant fraction discriminator (CFD). The CFD is used to discriminate fission fragment pulses from alpha particle pulses. The output of the CFD is sent to a resistor-capacitor (RC) filter to shape the NIM pulse from the CFD. The RC filter is an integrator that integrates the NIM pulse using a selectable time constant and adjustable gain. The RC filter is used for pulse mode operation of detectors. The signal is then sent to the Fourier analyzer where the signal is digitized.

The detector signals are processed in much the same way as the source signal. As an example consider the use of ${ }^{3} \mathrm{He}$ proportional chambers in a subcritical noise measurement. The signals from the ${ }^{3} \mathrm{He}$ detectors are sent to a preamplifier and then to a linear amplifier (AMP). A high voltage power supply is used to power the chamber. The linear amplifier time constants are typically selectable. The linear amplifier output is sent to a single-channel analyzer (SCA). The SCA is used to reject unwanted pulses or pulses due to gamma ray pileup and produces a logic (TTL) pulse. The SCA output is directed to a TTL-to-NIM converter and then to the RC filter prior to being sent to the Fourier analyzer. The TTL-to-NIM converter is required because the RC filter only accepts NIM pulses. A sketch of the electronic configuration is provided in Fig. 7. Additional electronic configurations are presented in the dissertation by King. ${ }^{33}$

The majority of the components used in these measurements are commercially available. The high-gain fast amplifier for the source is currently manufactured at ORNL and by the RIS corporation. The ${ }^{252} \mathrm{Cf}$ source ionization chambers are currently manufactured at ORNL and in several Russian institutes. The electronic components are commercially available except for the RC filters that are manufactured at ORNL. The RC filters could easily be manufactured at other facilities because the design is not proprietary.

\subsection{FOURIER PROCESSOR AND COMPONENTS}

Fourier processors are commercially available and have found wide use in the nuclear industry. One of the most common applications is monitoring reactor core barrel vibrations in nuclear power plants. Several components are required for use with the Fourier processor. These components included filters and analog to digital converters. Many commercially available Fourier processors have these components built into the system making selection of such devices unnecessary. The filters are required to prevent aliasing ${ }^{15}$ from occurring due to the digitization of the continuous signal. Aliasing occurs if the digitized signal is not sampled at least twice the frequency of the maximum frequency in the signal. For example, if the maximum frequency is $10 \mathrm{kHz}$ then the signal must be sampled at least $20 \mathrm{kHz}$ to avoid aliasing. 

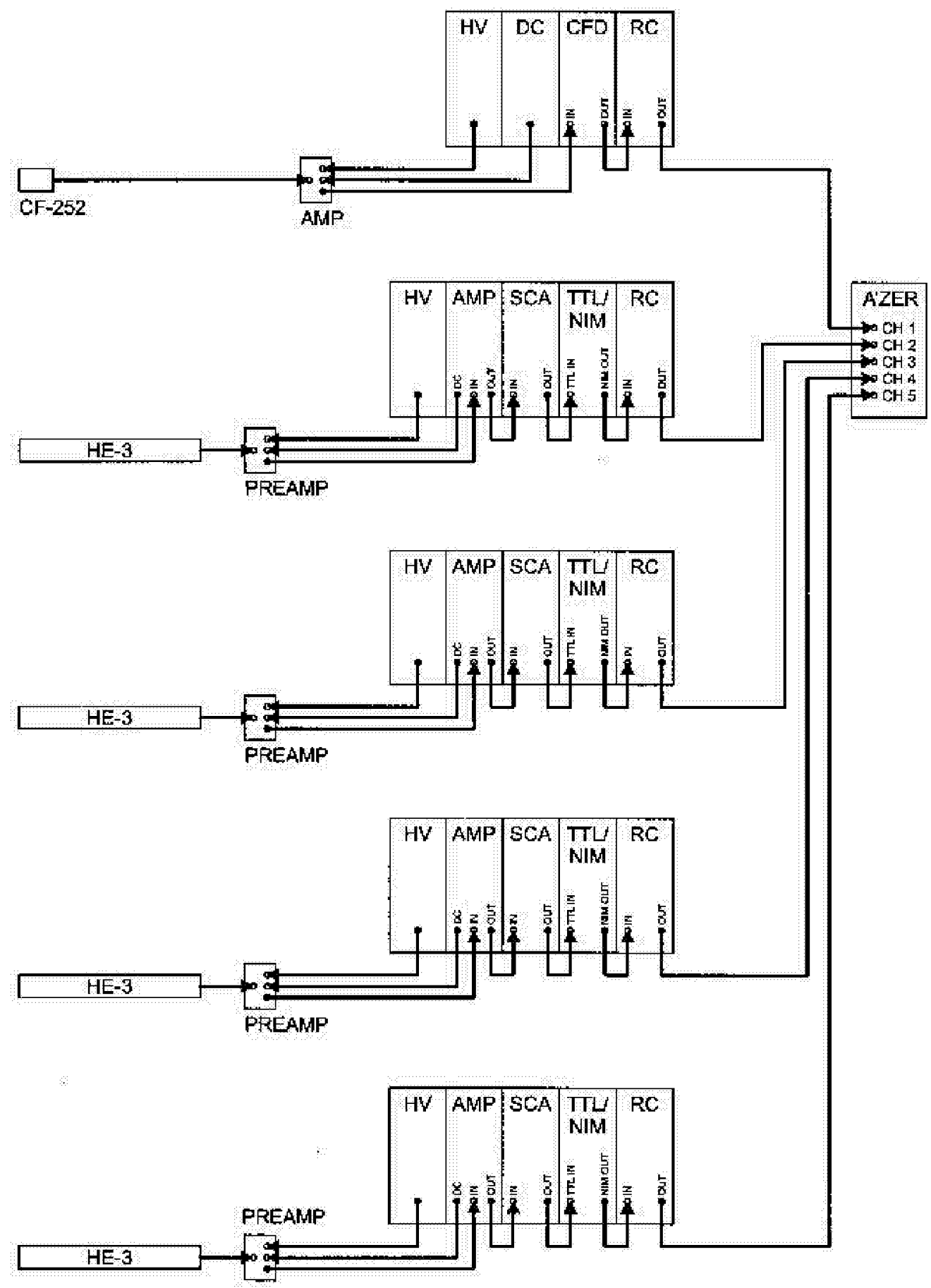

Fig. 7. Typical detection system configuration. 
A low-pass filter is commonly used to avoid aliasing of discrete time signals. The low pass filter essentially eliminates frequencies greater than the maximum frequency in the signal. A response window is usually applied to the discrete time series to reduce the leakage of adjacent frequency bins. The implementation of the discrete Fourier transform assumes that the discrete signal is periodic. However, this is not the case for subcritical noise measurements. Therefore, appropriate window functions are used to minimize the leakage errors. Such window functions are also commonly built into the Fourier processor. The Fourier processor also requires the use of an analog-to-digital (A/D) converter. The A/D converter digitizes the signal from the filters. The signal from the filter must have sufficient amplitude to be digitized. The common requirements for the Fourier processor include selectable block size, selectable sampling rates, batch mode operation, and minimal cross talk between channels. Cross talk between channels is very important for low coherence measurements. Fourier processors that meet these requirements are available. One such processor is the WCA processor developed by ZONIC Corporation. This analyzer has a variable sampling rate from 2.5 to $100 \mathrm{kHz}$ and selectable block size from 64 to 2048 points. The WCA processor is portable and uses a personal computer to store the acquired auto and cross spectra as can be seen in the photograph in Fig. 8. This analyzer is currently used by ORNL for subcritical noise measurements.

For systems that have a frequency response greater than $100 \mathrm{kHz}$, a special processor can be developed. Such processors have been developed at ORNL that allow operation up to $32 \mathrm{MHz}$ and up to $1 \mathrm{GHz}$. The $32-\mathrm{MHz}$ processor is no longer in operation. The $1 \mathrm{GHz}-$ processor is limited to sparse counting operation. This processor actually performs measurements in the time domain by taking advantage of the sparse number of counts per data block. The processor performs correlation calculations by only allowing a single count to occur in a time bin. The correlation functions between signals are calculated by constructing a histogram of the time-difference between detector signals for each data block. This processor should not be used for high-count rate situations that could cause more than one count in a single time bin. The technology for developing processors with sampling rates greater than $100 \mathrm{kHz}$ exists and could probably be obtained from commercial enterprises. 


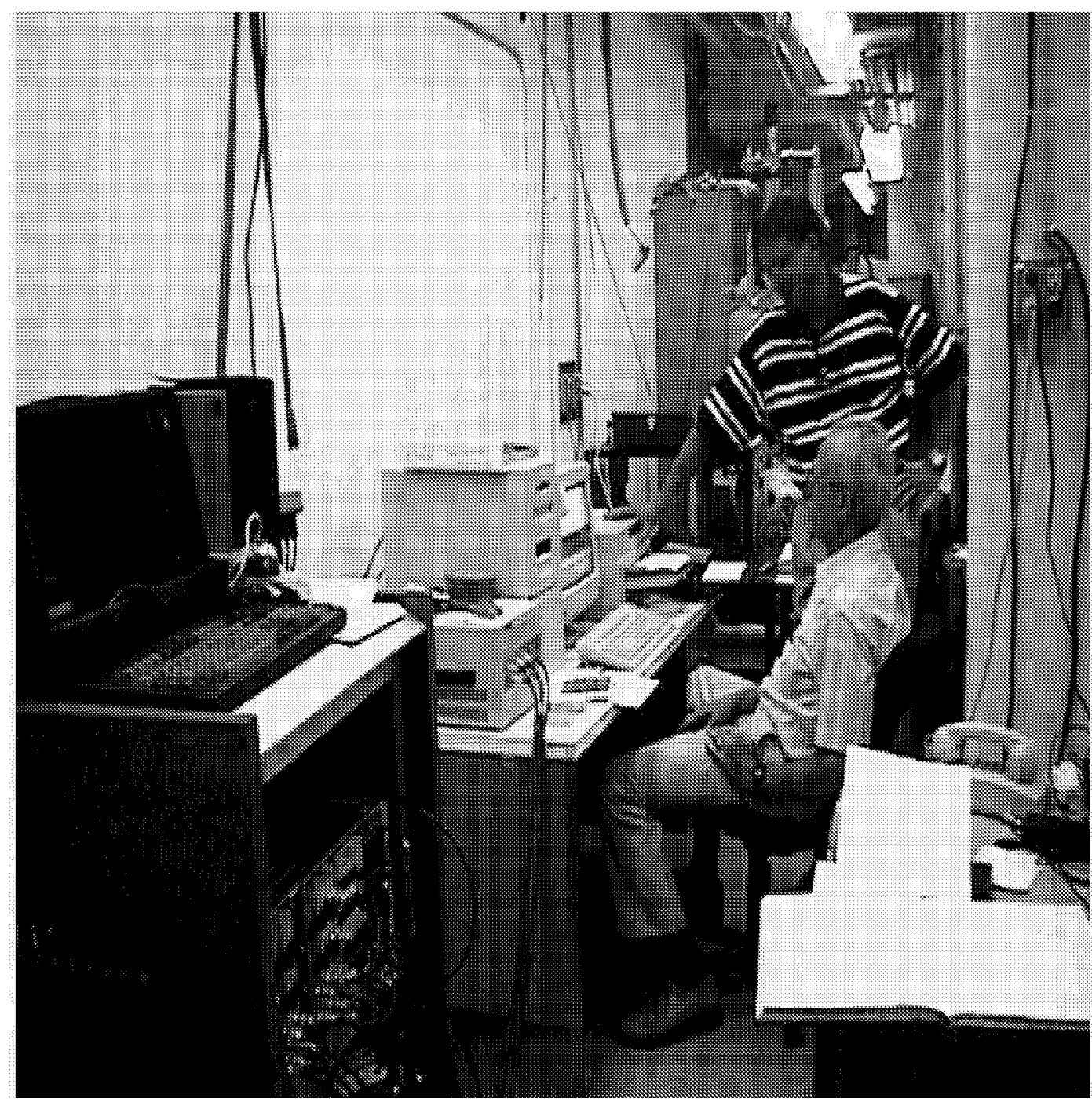

Fig. 8. Photograph of ZONIC WCA Fourier processor. 


\section{CHAPTER 4}

\section{APPLICATION OF SUBCRITICAL NOISE MEASUREMENTS}

Subcritical noise measurements have been performed for a variety of fissile systems. The implementation of the measurement depends on the particular application. Perhaps the most useful application would be use of this measurement to benchmark nuclear data and Monte Carlo codes. Additionally, subcritical noise measurements can be used for in-situ measurements of configurations of nuclear material. Other applications could include process monitoring in which the interpretation of the measurement may not be necessary.

\subsection{REVIEW OF SUBCRITICAL NOISE MEASUREMENTS}

A variety of measurements have been performed by ORNL at various facilities. Measurements have been performed with uranium, plutonium, and mixtures of uranium and plutonium. Fissile system configurations have varied greatly and include arrays or light water reactor fuel assemblies, spheres, cylinders, tanks, interacting cylinders, interacting tanks, annuli, and other arrays. Fissile materials include both liquid and metal systems with fuel types of oxides, fluorides, nitrates, and metals. The enrichment of the systems has varied from $2.5 \mathrm{wt} \%$ to $93 \mathrm{wt} \%{ }^{235} \mathrm{U}$. Measurements have been performed for initial loading of reactors, quality assurance of reactor fuel elements, fuel preparation and processing facilities, and zero power testing of reactors. A brief description of these experiments is provided in Table 4.1 .

Table 4.1. Summary of subcritical noise measurements

\begin{tabular}{|c|c|c|c|}
\hline Experiment & Facility & Date & Features \\
\hline $\begin{array}{l}\text { 1. FFTF Reactor } \\
\text { Mockup }^{34}\end{array}$ & $\begin{array}{l}\text { ZPR-9 } \\
\text { Argonne }\end{array}$ & 1974 & $\begin{array}{l}\text { Fast breeder reactor mockup with } \\
540 \mathrm{~kg} \mathrm{Pu} \text { metal }\end{array}$ \\
\hline $\begin{array}{l}\text { 2. Uranium metal } \\
\text { sphere and } \\
\text { cylinders }^{34}\end{array}$ & $\begin{array}{l}\text { Y-12 Critical } \\
\text { Facility }\end{array}$ & 1975 & $\begin{array}{l}\text { Unmoderated and unrelfected } \\
\text { HEU metal }\end{array}$ \\
\hline $\begin{array}{l}\text { 3. Water moderated } \\
\text { research reactor }\end{array}$ & $\begin{array}{c}\text { Pool critical } \\
\text { facility ORNL }\end{array}$ & 1978 & $\begin{array}{l}\text { Plate type water moderated and } \\
\text { reflected research reactor }\end{array}$ \\
\hline $\begin{array}{l}\text { 4. Fuel solution } \\
\text { cylinder }^{36}\end{array}$ & $\begin{array}{l}\text { TA-18 } \\
\text { LANL }\end{array}$ & 1980 & $\begin{array}{l}4.95 \mathrm{wt} \%{ }^{235} \mathrm{U} \text { uranyl fluoride } \\
\text { solution }\end{array}$ \\
\hline $\begin{array}{l}\text { 5. Fuel solution } \\
\text { cylinder }^{37}\end{array}$ & $\begin{array}{l}\text { PNL Critical } \\
\text { Facility }\end{array}$ & 1981 & $\begin{array}{l}\text { Unreflected mixed Pu-uranyl } \\
\text { nitrate }\end{array}$ \\
\hline $\begin{array}{l}\text { 6. HFIR fuel } \\
\text { element }^{38}\end{array}$ & ORNL & 1982-84 & $\begin{array}{l}\text { Annular fuel plates moderated } \\
\text { and reflected with water }\end{array}$ \\
\hline $\begin{array}{l}\text { 7. Fuel solution } \\
\text { cylinder }^{39}\end{array}$ & ORNL & 1983 & $\begin{array}{l}\text { Unreflected uranyl nitrate with } \\
\text { densities } 0.3 \text { to } 13.7 \mathrm{~g} \mathrm{~g}{ }^{235} \mathrm{U} / \ell\end{array}$ \\
\hline 8. LWR fuel pins ${ }^{40}$ & $\begin{array}{l}\text { B\&W critical } \\
\text { facility }\end{array}$ & 1983 & $\begin{array}{l}\text { 300-5000 LWR fuel pins with } \\
1500-4000 \mathrm{ppm} \text { boron }\end{array}$ \\
\hline
\end{tabular}


Table 4.1. Summary of subcritical noise measurements (Cont'd.)

\begin{tabular}{|c|c|c|c|}
\hline Experiment & Facility & Date & Features \\
\hline $\begin{array}{l}\text { 9. Uranium metal } \\
\text { cylinders }{ }^{41}\end{array}$ & ORNL & 1984 & $\begin{array}{l}\text { HEU metal cylinders 7-in. OD, 2- } \\
\text { 4-in. thick }\end{array}$ \\
\hline $\begin{array}{l}\text { 10. Interacting } \\
\text { uranium metal } \\
\text { cylinders }\end{array}$ & ORNL & 1984 & $\begin{array}{l}\text { Two unreflected HEU cylinders } \\
\text { separated by air and boraplaster }\end{array}$ \\
\hline 11. HEU safe ${ }^{43}$ & $\mathrm{Y}-12$ & 1984 & HEU metal slabs in storage safe \\
\hline $\begin{array}{l}\text { 12. Interacting } \\
\text { solution tanks }\end{array}$ & ORNL & 1985 & $\begin{array}{l}\text { Two 7.5-in. OD, } 35 \text {-in. tall } \\
\text { cylinders with uranyl nitrate } \\
\text { solution }\end{array}$ \\
\hline $\begin{array}{l}\text { 13. Interacting } \\
\text { solution storage } \\
\text { bottles }\end{array}$ & $\overline{\text { ORNL }}$ & 1985 & 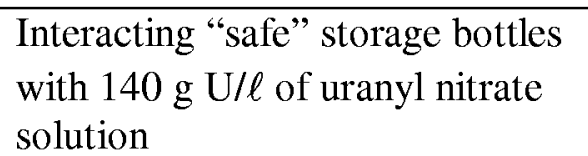 \\
\hline $\begin{array}{l}\text { 14. Fuel solution } \\
\text { cylinder }{ }^{6}\end{array}$ & ORNL & 1985 & $\begin{array}{l}\text { Static and dynamic measurements } \\
\text { with HEU uranyl nitrate solution } \\
\text { in a 10-in OD solution tank }\end{array}$ \\
\hline 15. Annular tank ${ }^{4 /}$ & PNL & 1985 & $\begin{array}{l}\text { Unreflected, plutonium-uranyl } \\
\text { nitrate solution with varying } \\
\text { heights }\end{array}$ \\
\hline 16. Slab tank ${ }^{48}$ & PNL & 1985 & $\begin{array}{l}\text { Unreflected, plutonium-uranyl } \\
\text { nitrate solution with varying } \\
\text { height and thickness }\end{array}$ \\
\hline $\begin{array}{l}\text { 17. Reactor } \\
\text { mockup }^{49}\end{array}$ & ANL-West & 1998 & $\begin{array}{l}\text { SP-100 reactor mockup for } \\
\text { various core loadings }\end{array}$ \\
\hline $\begin{array}{l}\text { 18. Interacting slab } \\
\text { tanks }\end{array}$ & $\begin{array}{l}\text { TA-18 } \\
\text { LANL } \\
\end{array}$ & 1989 & $\begin{array}{l}\text { Interacting slab tanks with HEU } \\
\text { uranyl nitrate solution }\end{array}$ \\
\hline $\begin{array}{l}\text { 19. Uranium hydride } \\
\text { cylinders }\end{array}$ & $\begin{array}{l}\text { TA-18 } \\
\text { LANL }\end{array}$ & 1989 & $\begin{array}{l}\text { HEU-hydride cylinders with } \\
\text { varying heights }\end{array}$ \\
\hline $\begin{array}{l}\text { 20. Plutonium } \\
\text { annuli }^{52}\end{array}$ & $\begin{array}{l}\text { TA-55 } \\
\text { LANL }\end{array}$ & 1993 & $\begin{array}{l}{ }^{242} \mathrm{Pu} \text { annuli with polyethylene } \\
\text { moderators }\end{array}$ \\
\hline $\begin{array}{l}\text { 21. HEU storage } \\
\text { vault }^{29}\end{array}$ & Y-12 & 1993 & $\begin{array}{l}\text { Array of HEU metal castings in } \\
\text { concrete storage vault }\end{array}$ \\
\hline $\begin{array}{l}\text { 22. Research reactor } \\
\text { fuel elements }\end{array}$ & $\begin{array}{l}\text { University of } \\
\text { Missouri }\end{array}$ & 1998 & $\begin{array}{l}\text { Array of water moderated HEU } \\
\text { research reactor fuel elements } \\
\text { with various absorbers }\end{array}$ \\
\hline
\end{tabular}

Many of these experiments have only been documented in proceedings for professional society meetings and have not been documented in laboratory or journal reports. A detailed analysis of these experiments should be pursued. Subcritical experiments with loosely and tightly coupled systems can provide a significant amount of data for benchmark purposes. Likewise, experiments with plutonium-uranyl nitrate solutions 
systems also provide data that can be used for benchmark purposes. The final measurement in Table 4.1 is a benchmark experiment that was performed at the University of Missouri by ORNL and was analyzed by Westinghouse Safety Management Solution Systems for Savannah River Technology Center.

\subsection{SUBCRITICAL BENCHMARK MEASUREMENTS}

A variety of measurements listed in Table 4.1 can serve as subcritical benchmarks. Many of these measurements have been performed in critical experiment facilities and research reactors. The configurations and materials for many of these systems are well known and could be simulated using Monte Carlo models. Measurements performed with uranyl nitrate solutions, mixed uranyl and plutonium nitrate, research reactor fuel elements, and PWR fuel pins provide data that can be used for cross section testing and validation of Monte Carlo codes. The subcritical measurements cover $k_{\text {eff }}$ values varying from 0.3 to 0.99 . The uncertainty on the measured $k_{\text {eff }}$ values are typically less than one percent for $k_{\text {eff }}$ values greater than 0.6. Although the uncertainty could become larger than one percent for $k_{\text {eff }}$ values less than 0.6 , far subcritical measurements can still provide useful data for benchmark purposes. Some of these measurements will be evaluated for the International Criticality Safety Benchmark Evaluation Project (ICSBEP).

The subcritical noise measurement has several advantages over other subcritical measurement techniques for benchmarking nuclear data and Monte Carlo codes. One of the primary factors is that the spectral ratio obtained from the noise measurement does not depend on detection efficiency. This is a significant advantage in that a change in the detection system would not manifest itself in the spectral ratio. Another distinct advantage is that the prompt neutron decay constants can also be obtained from this measurement. In principle, subcritical noise measurements should be performed in lieu of Rossi- $\alpha$ measurements because multiple quantities are available from the noise measurements. Although the spectral ratio depends on knowing the ratio of the inherent spontaneous fission rate to the ${ }^{252} \mathrm{Cf}$ spontaneous fission rate for systems with large inherent sources, the inherent fission rate would be known for benchmark measurements.

The reproducibility of the measurement results over long periods of time has been demonstrated experimentally. The reproducibility is demonstrated with measurements with uranium metal cylinders performed first in 1975 and repeated in 1984. The spectral ratio vales are statistically the same for these two sets of measurements although different

${ }^{252} \mathrm{Cf}$ sources and detectors were used in the measurements. The reproducibility of the measurement is necessary if the method is to be used for benchmark purposes.

Another advantage is that quantities from the subcritical noise measurement have high sensitivity to small changes in configurations of fissile materials. This sensitivity has been observed experimentally and demonstrated with Monte Carlo simulations ${ }^{54}$. The high sensitivity of the noise quantities to small changes in fissile configurations makes the measurement ideal for testing differences in nuclear cross section data sets. The spectral ratio is proportional to reactivity as shown in Chap 2. Therefore, for a small 
change in $k_{\text {eff }}$ a greater change would occur in the spectral ratio. This enhanced sensitivity would make it easier to evaluate the effect of small changes in nuclear cross sections and neutron multiplicity data. This enhanced sensitivity leads to the extension of these measurements to high-order spectral measurements. These higher order signatures have even greater sensitivity and should be investigated in more detail.

\subsection{IN-SITU SUBCRITICALITY MEASUREMENTS}

The second area of applicability of subcritical noise measurements is for in-situ determinations of the subcritical neutron multiplication factor. The two ways that this can be achieved is by post analysis of the measurements using the Monte Carlo method previously described or by using the limited point reactor kinetics models to relate the measured quantity to the subcritical neutron multiplication factor. The latter method should only be considered if an on-line determination of $k_{\text {eff }}$ is desired. In such applications, care must be taken to properly select the location of the source and detectors such that the point kinetics models are applicable. Furthermore, calculations of spatial correction factors and importance ratios are also required and must be supplied to the point reactor formulas to determine $k_{\text {eff. }}$. The use of point reactor kinetics is discouraged because uncertainties in correction factors contribute directly to the uncertainty in the reactivity estimates. The Monte Carlo method is the preferred way to interpret the measurements because of the limitations of the point reactor models and because correction factors do not have to be computed.

In-situ subcriticality measurements performed at the Oak Ridge Y-12 Plant in Vault 16 demonstrated that these types of measurements are practical ${ }^{29}$. In these measurements, the subcriticality of an array of high-enriched uranium metal castings inside a concrete storage vault was determined using the Monte Carlo method. Measurements were performed with the source and detectors placed inside the array. The source was located in the center of the central casting and the detectors were placed on the periphery of the array. Prior to performing the noise measurements, flux profile measurements were performed to determine the essentially infinite array size. The detectors were placed on the outside of the essentially infinite array. The noise measurements were performed over many weeks to obtain statistically converged spectra because of the low coherence values. The measurements were interpreted using MCNP-DSP simulations. These simulations were also performed over several weeks. The Monte Carlo models contained as much detail as possible about the storage vault configuration; however, the composition of the concrete storage vault was not known. The analyses were performed assuming that the concrete was dry or wet. The interpreted $k_{\text {eff }}$ value obtained from the analyses did not depend on the exact knowledge of the concrete composition. Although the concrete composition was not exactly known, the concrete vault was 25 years old and should have been comprised of dry concrete. Coincidentally, the calculated spectral ratio value for the dry vault agreed within one standard deviation of the measured value. The Monte Carlo models included as much detail as possible concerning the storage vault geometry and composition. The storage trays were modeled in great detail, as were the uranium metal castings. The composition of the storage trays, detectors, and the castings 
were well known and included in the models. Although the interpreted $k_{\text {eff }}$ value for the HEU storage vault does not depend on the composition of the concrete, all other details about the measurement are known. The success of the interpretation of the HEU storage vault measurements occurs because the spectral ratio depends only on the induced fission events from the ${ }^{252} \mathrm{Cf}$ source. A significant inherent neutron source does not exist in this configuration.

Systems that include a significant inherent neutron source can behave differently. The spectral ratio is dependent on the induced fission events from the ${ }^{252} \mathrm{Cf}$ source and any inherent neutron sources. To interpret the measurements, the inherent neutron source contribution can be measured. Passive noise measurements without the ${ }^{252} \mathrm{Cf}$ source can be performed to obtain an estimate of the contribution of the inherent neutron source. Because the inherent neutron sources only affect the detector-detector cross spectra (see in Chap. 2), a passive measurement of the detector-detector cross spectra provides a measured contribution of the inherent source. The passive measurement would be used to determine the ratio of the ${ }^{252} \mathrm{Cf}$ induced fission contribution to the inherent neutron source. This information would be used in the Monte Carlo calculations to determine the subcritical neutron multiplication factor by including an inherent source. This inherent source could either be a spontaneous fission source such as ${ }^{244} \mathrm{Cm}$ or ${ }^{240} \mathrm{Pu}$ or could be a result of $\alpha-n$ reactions in the system. These inherent sources must be included in the Monte Carlo calculations to determine $k_{\text {eff. }}$ For systems with both unknown inherent spontaneous fission and $\alpha$-n sources, the determination of $k_{\text {eff }}$ becomes more complicated because the relative effect of each source cannot be determined by measurements. Therefore, additional information would be required to determine the relative amounts of $\alpha-n$ to inherent spontaneous fission sources. A modification to the current measurement technique has been developed to obtain a similar spectral ratio that does not depend on inherent neutron sources. This extension to the current measurement will be briefly discussed in Chap. 5.

Several issues must be addressed before performing an in-situ or a benchmark subcritical noise measurement. The "infinite" array size of the system must be determined. This may be a concern for storage arrays of spent nuclear fuel in that one spent fuel assembly in a storage pool may not communicate neutronically with another element that is far removed. Therefore, measurements would only be performed on sections of arrays that communicate neutronically with each other as determined by measurement. Furthermore, the selection of the source size will be dependent on the application. For systems with large inherent sources, it is important that a large source or multiple sources be used. In cases where the inherent neutron source is not known, the maximum credible inherent source size should be used. A recommended source size would be approximately $5 \%$ of the inherent source size. This source could be a single source or comprised of multiple source ionization chambers. Source and detector locations must be carefully determined if point reactor kinetics models are to be used. The source and detectors should be sufficiently separated to minimize direct source neutrons from interacting with the detectors when possible. Likewise, the detectors should be sufficiently separated to "view" more of the fissile system. Additionally, the detector time response must be shorter than the system time response if frequency spectra are to be fitted to determine 
the prompt neutron decay constants. A final but important requirement is that the detection efficiency must be properly selected to perform the measurements in the required time frame. The measurement time may not be a limiting factor for storage conditions; however, if the measurement time is short, a detector with high efficiency must be used. Furthermore, environmental factors will also affect the selection of the detectors to be used for in-situ or benchmark measurements.

\subsection{PROCESS MONITORING}

Another useful application of subcritical noise measurements is to provide a way to monitor fissile material processing operations. This application would not focus on determining the subcritical neutron multiplication but would use the measured quantities as a way to ascertain normal operation. The enhanced sensitivity of the subcritical noise measurement would allow fissile processing operations to be monitored and controlled using the changes in spectral signatures as indicators to changes in the system. The spectral ratio is an ideal candidate for process monitoring because it is independent of detection efficiency and would not change due to drifts in the electronic system. Measurements and Monte Carlo simulations of a process condition could be performed to provide bounding values for the spectral ratio. The measured spectral ratio values could be equated to $k_{\text {eff }}$ values for various operating conditions. Changes in the spectral ratio value could be monitored and used to ensure that the reactivity of the system remains below some desired value. This would enhance safety in operations with fissile material by providing some means to estimate the subcriticality of the fissile process. 


\section{CHAPTER 5}

\section{EXTENSIONS OF SUBCRITICAL NOISE MEASUREMENTS}

Extensions to any measurement method are to be expected with advances in data acquisition systems and data analysis techniques. This chapter briefly describes two possible extensions that could make the measurement more generally applicable to in-situ measurements. These extensions could also be used for benchmark measurements in addition to the standard source-driven noise measurement.

\subsection{ALTERNATE NEUTRON SOURCES}

Currently, source-driven noise analysis measurements are performed using a spontaneous fission source in an ionization chamber. However, this method does not have to be limited to spontaneous fission sources. A neutron generator (D-D or D-T) could be used to replace the spontaneous fission source. Several advantages are manifested using neutron generators that operate in pulse mode. First, the spectral ratio defined by Eqs. 2.35 and 2.36 would have less dependence on the neutron source. The detector-detector cross spectra would not include a term that depends on the second reduced moment of the source multiplicity distribution $\overline{n_{0}\left(n_{0}-1\right)}$. This term would be zero for pulsed neutron sources because only one neutron per event is generated. Furthermore, the direction of the neutron can be determined using an associated particle source. In the D-T reaction, an alpha particle is produced that can be detected using a scintillation detector. The signal of the photo-multiplier tube could serve as the time signal for neutrons emitted in a defined direction from the neutron generator. Unlike the spontaneous fission sources the neutron generators can be turned off and thus would reduce personnel exposure.

\subsection{BI-SPECTRAL RATIO MEASUREMENT}

Perhaps the most significant extension of the source-driven subcritical measurement would include the measurement of the bi-spectral ratio. The bi-spectral ratio is closely related to the standard spectral ratio; however, the bi-spectral ratio involves only events correlated with the timed neutron source. The summary provided in this section follows directly from the developments of Mattingly ${ }^{11}$. The bi-spectral ratio is defined as

$$
\Psi_{X Y \mid S}=\frac{S_{S X}(0) S_{S Y}(0)}{\bar{s} S_{S X Y}(0,0)}=\frac{\overline{n_{0}}}{\frac{\bar{n}_{0}\left(n_{0}-1\right)}{\overline{n_{0}}}+\frac{\overline{n(n-1)}}{\bar{n}|r|}} .
$$


The bi-spectral ratio is essentially equivalent to the standard spectral ratio for systems but is independent of the source detection efficiency, the detector efficiency, or an inherent source. This measurement requires pulse neutron measurements to be performed with two detectors. In addition, the bi-spectral ratio requires the correlation of the response of two detectors with the neutron source. This measurement can be thought of as a dual pulse neutron measurement. This measurement is only dependent on fission chains initiated by the neutron source and hence would be more generally applicable to in-situ measurements with plutonium and irradiated nuclear fuels. For systems close to delayed critical, the standard spectral ratio will be dependent on the delayed neutrons that will behave as an inherent neutron source. However, the bi-spectral ratio will not be affected by delayed neutrons and hence would be more useful for systems close to delayed critical. In theory, the bi-spectral ratio can be measured up to prompt critical.

The bi-spectral ratio was derived from a high-order correlation measurement between the timed neutron source and two detectors. The integral time-domain expression for the bispectral ratio is

$$
\Psi_{X Y \mid S}=\frac{\int d t C_{S X}(t) \int d t C_{S Y}(t)}{s \int d t_{1} \int d t_{2} C_{S X Y}\left(t_{1},{ }_{2}\right)} .
$$

$C_{s x y}\left(t_{l}, t_{2}\right)$ is the bi-correlation between the source and two detectors, and $\bar{s}$ is the average source fission rate. The expression in Eq. 5.2 can be thought of as the ratio of two-way coincident events between two pairs of channels divided by a three-way coincident event that is normalized to the source fission rate. 


\section{CHAPTER 6}

\section{SUMMARY}

The subcriticality of fissile materials is essential to safe processing, transportation, and storage operations. The assurance of the subcriticality of various fissile material operations is commonly obtained by detailed criticality safety evaluations for the specific operation. The criticality safety evaluations are performed using detailed computations that have been validated using critical experiments in which the neutron multiplication factor is one. In most cases, the actual fissile material operation does not coincide to the conditions that exist for the critical experiment, i.e. the geometry and neutron spectrum may differ between the subcritical operation and the critical experiment. These differences require that additional safety margins be included in the criticality safety evaluations. Subcritical measurements can be used to address the use of additional safety margins by providing supplementary benchmark data for validating computation methods or by providing an in-situ measurement of the degree of subcriticality. The use of subcritical measurements would actually enhance the safety of operations with fissile materials.

Subcritical source-driven noise measurements are simultaneous Rossi- $\alpha$ and randomly pulsed neutron measurements that provide measured quantities that can be related to the subcritical neutron multiplication factor. Point reactor kinetics models can be used to relate the measured quantities to the subcritical neutron multiplication factor. More elaborate models can also be developed using a generalized stochastic model. These measurements can also be simulated using Monte Carlo codes to determine the subcritical neutron multiplication factor or to determine the sensitivity of calculations to nuclear cross section data. The interpretation of the measurement using a Monte Carlo method is based on a perturbation model for the relationship between the spectral ratio and the subcritical neutron multiplication factor. The subcritical source-driven noise measurement has advantages over other subcritical measurement methods in that reference measurements at delayed critical are not required for interpreting the measurements. Therefore, benchmark or in-situ subcritical measurements can be performed outside a critical experiment facility. Furthermore, because a certain ratio of frequency spectra has been shown to be unaffected by drifts or changes in instrumentation during the measurement, the spectral ratio is an ideal candidate for process monitoring.

Measurements have been performed by ORNL at various facilities with uranium, plutonium, and mixtures of uranium and plutonium. Fissile system configurations have varied greatly and include arrays or light water reactor fuel assemblies, spheres, cylinders, tanks, interacting cylinders, interacting tanks, annuli, and other arrays. Fissile materials include both liquid and metal systems with fuel types of oxides, fluorides, nitrates, and metals. The enrichment of the systems has varied from $2.5 \mathrm{wt} \%$ to $93 \mathrm{wt} \%$

${ }^{235} \mathrm{U}$. Several of these measurements could be used as benchmark measurements. 
An extension of the source-driven subcritical noise measurement has also been discussed that eliminates the few technical challenges for in-situ applications. This promising extension is the development of the bi-spectral ratio. The bi-spectral ratio has been theoretically shown to be independent of detection efficiency, source detector efficiency, and any inherent sources and is directly related to the subcritical reactivity. Measurements of the bi-spectral ratio should be performed to demonstrate the advantages of this measurement for systems with inherent neutron sources or that are close to delayed critical. 


\section{REFERENCES}

1. $\quad$ ANSI (American National Standards Institute), 1998. "Nuclear Criticality Safety in Operations with Fissionable Materials Outside Reactors," ANSI/ANS-8.11998, American Nuclear Society.

2. B. E. Simmons and J. S. King, "A Pulsed Neutron Technique for Reactivity Determination,” Nucl. Sci. Eng., 3, 595 (1958).

3. J. T. Mihlaczo, "The Use of ${ }^{252} \mathrm{Cf}$ as a Randomly Pulsed Neutron Source for Prompt Neutron Decay Measurements," Nucl. Sci. Eng., 41, 296 (1970).

4. J. D. Orndoff, "Prompt Neutron Periods of Metal Critical Assemblies," Nucl. Sci. Eng., 2, 450 (1957).

5. F. J. Jankowski, D. Klein, and T. M. Miller, "Calibration of Control Rods," Nucl. Sci. Eng., 2, 288 (1957).

6. C. A. Sastre, "The Measurement of Reactivity," Nucl. Sci. Eng., 8, 443 (1960).

7. N. J. Ackermann, Jr., "Subcriticality Measurements in an LMFBR," Nuclear Safety, 12, 587 (1971).

8. V. K. Paré and J. T Mihalczo, "Reactivity from Power Spectral Density Measurements with Californium-252," Nucl. Sci. Eng. 56, 213 (1975).

9. A. F. Henry, Nuclear Reactor Analysis, Massachusetts Institute of Technology, Cambridge, Massachusetts (1975).

10. E. P. Ficaro and D. K. Wehe, "Monte Carlo Simulations of the ${ }^{252} \mathrm{Cf}-$ Source-Driven Noise Analysis Measurements for Determining Subcriticality," Proceedings International Topical Meeting Advances in Mathematics, Computations and Reactor Physics, Pittsburgh, Pennsylvania, April 28-May 2, 1991, Vol. 1, p. 5.22.1, American Nuclear Society, 1991.

11. J. K. Mattingly, "High Order Statistical Signatures from Source-Driven Measurements of Subcritical Fissile Systems," Ph.D. Dissertation, University of Tennessee, Knoxville, Tennessee, August 1998.

12. R. P. Feynman, F. De Hoffmann, R. Serber, J. Nuclear Energy, 3, 64 (1956).

13. G. R. Keepin, Physics of Nuclear Kinetics, Addison-Wesley Publishing Co. Inc., Reading, Massachusetts, (1965). 
14. R. E. Uhrig, Random Noise Techniques in Nuclear Reactor Systems, Ronald Press Co., New York, New York (1970).

15. A. V. Oppenheim and R. W. Shafer, Digital Signal Processing, Prentice-Hall, Inc., Englewood Cliffs, New Jersey (1975),

16. N. G. Van Kampen, Stochastic Processes in Physics and Chemistry, Elseiver Science Publishers, Amsterdam, The Netherlands (1992).

17. W. M. Stacey, Jr., Space-Time Nuclear Reactor Kinetics, Academic Press, Inc., New York, New York (1969).

18. J. L. Munoz-Cobo, R. B. Perez, and G. Verdu, Nucl. Sci. Eng., 95, 83 (1987).

19. J. L. Muñoz-Cobo and G. Verdu, "Neutron Stochastic Transport Theory with Delayed Neutrons," Annals Nuclear Energy 14, 7,327 (1987).

20. J. L. Munoz et al., Proceedings of the International Conference on Mathematics and Computations, September 1999, Madrid, Spain.

21. M. M. R. Williams, Random Processes in Nuclear Reactors, Pergamon Press, Oxford, United Kingdom (1974).

22. I. Lux and L. Koblinger, Monte Carlo Particle Transport Methods: Neutron and Photon Calculations, CRC Press, Inc., Boca Raton, Florida (1991).

23. E. P. Ficaro, KENO-NR: A Monte Carlo Code Simulating the ${ }^{252}$ Cf-Source-Driven Noise Analysis Experimental Method for Determining Subcriticality, Ph.D. Dissertation, University of Michigan, 1991.

24. L. M. Petrie, and N. F. Landers, "KENO V.a: An Improved Monte Carlo Criticality Program with Supergrouping," ORNL/NUREG/CSD-2, Oak Ridge National Laboratory, 1984.

25. T. E. Valentine and J. T. Mihalczo, "MNCP-DSP: A Neutron and Gamma Ray Monte Carlo Calculation of Source-Driven Noise-Measured Parameters," Ann. Nucl. Eng. 23, 16, 1271 (1996).

26. T. E. Valentine, "MCNP-DSP Users Manual”, ORNL/TM-13334, Oak Ridge Nat. Lab, January 1997.

27. J. F. Briesmeister, ed., MCNP4A-A General Monte Carlo N-Particle Transport Code, La-12625-M, Los Alamos National Laboratory, 1993. 
28. R. B. Perez, T. E. Valentine, J. T. Mihalczo, and J. K. Mattingly, "Determination of the Multiplication Factor and Its Bias by the ${ }^{252} \mathrm{Cf}$-Source Technique: A Method for Code Benchmarking at Subcritical Configurations," Proc. Topical Meeting of the Am. Nucl. Soc., Saratoga, N. Y. (1997).

29. J. T. Mihalczo, T. E. Valentine, and L. D. Phillips, "Subcritical Measurements for a HEU Storage Vault at the Y-12 Plant Using the ${ }^{252}$ Cf-Source-Driven Frequency Analysis Method," Nuclear Safety, 38, 1, January-March 1997.

30. T. E. Valentine, "Unreflected High-Enriched Uranyl Nitrate Solution Subcritical Noise Measurements," ICSBP Handbook (1999).

31. J. A. Williams, J. T. Mihalczo, C. W. Ricker, F. L. Glesius, and T. A. Kniss, "A High-Sensitivity, Position-Sensitive Fission Counter for Subcriticality Measurements of Spent Fuel," Nucl. Instrum. Meth. in Phys. Rev., A299, 187-90 (1990).

32. J. T. Mihalczo, P. E. Koehler, T. E. Valentine, and L. D. Phillips, Source Options for NWIS, ORNL/TM-13025, Martin Marietta Energy Systems, Inc., Oak Ridge National Laboratory (1995).

33. W. T. King, "Feasibility of ${ }^{252} \mathrm{Cf}$ Source-Driven Neutron Noise Measurements in Water Moderated Reactor," Ph.D. Dissertation, University of Tennessee, Knoxville, Tennessee (1980).

34. J. T. Mihalczo, V. K. Paré, G. L. Ragan, M. V. Mathis, and G. C. Tillett, "Determination of Reactivity from Power Spectral Density Measurements with Californium-252," Nucl. Sci. Eng., 66, 29-59 (1978).

35. W. T. King, J. T. Mihalczo, and E. D. Blakeman, "Preliminary Investigation of the Cf-252-Source Driven Noise Analysis Method of Subcriticality Measurement in LWR Fuel Storage and Initial Loading Applications," Trans. Am. Nucl. Soc., 47, 239-40 (1984).

36. J. T. Mihalczo, R. C. Kryter, and W. T. King, "Preliminary Investigation of ${ }^{252} \mathrm{Cf}$ Source-Driven Noise Analysis for Subcritical Fuel Solution System," Trans. Am. Nucl. Soc., 38, 359 (1981).

37. Unpublished experiment J. T. Mihalczo et al (1999).

38. J. T. Mihalczo and W. T. King, "Quality Assurance Verification of High-Flux Isotope Reactor Fuel Elements by the ${ }^{252} \mathrm{Cf}$-Source-Driven Noise Analysis Method," Nucl. Technol., 84, 205-23 (1988). 
39. J. T. Mihalczo, W. T. King, E. B. Johnson, and E. D. Blakemen, "Subcriticality Measurements for a Fuel Solution Tank with Changing Fuel Concentration Using Cf-252-Source-Driven Neutron Noise Analysis" Trans. Am. Nucl. Soc. 45, 337-38 (1984).

40. W. T. King, J. T. Mihalczo, and E. D. Blakeman, "Preliminary Investigation of the Cf-252-Source Driven Noise Analysis Method of Subcriticality Measurement in LWR Fuel Storage and Initial Loading Applications," Meeting of the American Nuclear Society, Washington, D.C., Trans. Am. Nucl. Soc. 47, 239-40 (1984).

41. J. T. Mihalczo, W. T. King, and E. D. Blakeman, "Subcriticality Measurements for Coupled Uranium Metal Cylinders Using the ${ }^{252} \mathrm{Cf}$-Source-Driven Neutron Noise Analysis Method," Nucl. Sci. Eng., 95, 1-13 (1987).

42. J. T. Mihalczo, W. T. King, and E. D. Blakeman, "Cf-252-Source-Driven Neutron Noise Analysis Measurements for Coupled Uranium Metal Cylinders," Meeting of the American Nuclear Society, Boston, Trans. Am. Nucl. Soc. 49, 241-43 (1985).

43. Unpublished experiment J. T. Mihalczo et al (1999).

44. J. T. Mihalczo, E. D. Blakeman, and W. T. King, "Subcriticality Measurements for Two Coupled Uranyl Nitrate Solution Tanks Using ${ }^{252}$ Cf-Source-Driven Neutron Noise Analysis Methods," Meeting of the American Nuclear Society, Reno, Nevada, Trans. Am. Nucl. Soc. 52, 640-42 (1986).

45. J. T. Mihalczo, E. D. Blakeman, and G. E. Ragan, “252 Cf-Source-Driven Neutron Noise Measurements for Three Interacting Tanks of Uranyl Nitrate Solution," Meeting of the American Nuclear Society, Dallas, June 7-11, 1987, Trans. Am. Nucl. Soc. 54, 208-9 (1987).

46. J. T. Mihalczo, E. D. Blakeman, G. E. Ragan, E. B. Johnson, and Y. Hachiya, "Dynamic Subcriticality Measurements Using the ${ }^{252} \mathrm{Cf}-$ Source-Driven Noise Analysis Method," Nucl. Sci. Eng., 104, 314-38 (1990).

47. J. T. Mihalczo, E. D. Blakeman, G. E. Ragan, R. C. Kryter, H. Seino, and R. C. Robinson, ${ }^{6252}$ Cf-Source-Driven Neutron Noise Measurements of Subcriticality for an Annular Tank Containing Aqueous Pu-U Nitrate," Nucl. Technol., 94, 336-60 (1991).

48. J. T. Mihalczo, E. D. Blakeman, G. E. Ragan, R. C. Kryter, H. Seino, and R. C. Robinson, ${ }^{4252}$ Cf-Source-Driven Noise Measurements of Subcriticality for a Slab Tank of Aqueous Pu-U-Nitrate," Nucl. Sci. Eng., 107, 35-57 (1991).

49. Unpublished experiment J. T. Mihalczo et al (1999). 
50. J. T. Mihalczo, E. D. Blakeman, V. K. Paré, T. E. Valentine, and D. L. Auslander, "Subcriticality of Two Uranyl Nitrate Flat Cylindrical Tanks Spaced in Air by the ${ }^{252}$ Cf-Source Driven Noise Analysis Method," Nucl. Technol., 103, 346-78 (1993).

51. Unpublished experiment J. T. Mihalczo et al (1999).

52. J. T. Mihalczo, V. K. Paré, E. D. Blakeman, and T. E. Valentine, "Time and Frequency Analysis Measurements for a ${ }^{242} \mathrm{Pu}$ Metal Ring Using ${ }^{252} \mathrm{Cf}$," Institute of Nuclear Materials Management 37th. Annual Meeting, Naples, Florida, July 1996.

53. T. E. Valentine, J. T. Mihalczo, R. C. Kryter, V. C. Miller, Subcritical SourceDriven Noise Analysis Measurements with Fresh and Spent Research Reactor Fuel Elements, ORNL/TM-13752, Oak Ridge National Laboratory, 1999.

54. J. T. Mihalczo and T. E. Valentine, "Calculational Verification and Process Control Applications Utilizing the High Sensitivity of Noise Measurement Parameters to Fissile System Configuration," Nucl. Sci. Eng., 121, 286-300 (1995). 
This page intentionally left blank. 


\section{APPENDIX A}

\section{SIMPLE THEORY OF FISSION CHAIN FLUCTUATIONS}

\section{A.1 POINT REACTOR KINETICS MODELS}

The simplest description of the time-dependent behavior of the neutron population in a subcritical system is best provided using the point reactor kinetics equations. This equation ignores the energy dependence and spatial dependence of the neutron population. The point reactor kinetics equation ${ }^{9}$ without delayed neutrons is defined as

$$
\frac{d}{d \mathrm{t}} n(\mathrm{t})+\frac{r}{\Lambda} \mathrm{n}(t)=S(t) .
$$

In this expression $n(t)$ is the time-dependent prompt neutron population, $S(t)$ is a timedependent neutron source, $r$ is the prompt reactivity, and $L$ is the neutron generationtime. The inclusion of the delayed neutrons is not necessary to describe the time evolution of the neutron population because in most subcritical systems the prompt neutron lifetime is typically several orders of magnitude smaller than the delayed neutron lifetime. In essence, the delayed neutrons behave as an inherent source that may produce fission neutrons that result in correlated detection events. The prompt neutron decay constant, $a$, is customarily defined as $a=r / L$ such that the point reactor kinetics equations are defined as

$$
\frac{d}{d \mathrm{t}} n(\mathrm{t})+\operatorname{an}(t)=S(t) .
$$

If a burst of neutrons is injected into a subcritical fissile system at time zero, the resulting prompt neutron fission chain will decrease exponentially as $e^{-a t}$. Assuming that the initial neutron population is zero, the time-dependent neutron population, $n$, behaves as

$$
n=\frac{S}{a}\left(1-e^{-a t}\right),
$$

following the injection of a source neutron, $S$, at time $t=0$. Only the fluctuations in the fission chains are observable in subcritical experiments. Therefore, the impulse response of interest would relate the fission rate to the source rate. The fission rate, $F(t)$, is defined as

$$
\bar{n} F(t)=\frac{n(t)}{\Lambda} .
$$

In this expression, $\bar{v}$ is the average number of neutrons from fission. The impulse response of the fission rate to the unit source would be defined as 


$$
h\left(t \mid t^{\prime}\right)=\frac{e^{-a\left(t-t^{\prime}\right)}}{\bar{n} \Lambda}
$$

This impulse response is interpreted as the expected number of fission events produced at time $t$ upon injection of a neutron at time $t^{\prime}$. This impulse response is used to describe the detection probability for chain-related events.

\section{A.2 NEUTRON DETECTION MODELS}

For practical purposes the following derivation of the detection events will only consider terminal detection events such as neutron capture. These detection models do not include the spatial or energy dependence of the detection process because the spatial dependence is treated as a point with a single energy group. The detector field-of-view and energy dependence will be represented by the detection efficiency that is defined as detector counts per fission. Hence, the efficiency provided in these models does not represent a measurable efficiency. The following derivations are similar to those developed by Ficaro ${ }^{10}$ and Mattingly ${ }^{11}$ in terms of the point kinetics equations previously defined.

The first part of the source-driven noise measurement to consider is the randomly pulsed neutron measurement. In a source-driven noise measurement, the source event is observable in time. The source could be either an instrumented spontaneous fission source or a pulse source such as a D-T or D-D source. The following derivation will assume that the source is a ${ }^{252} \mathrm{Cf}$ spontaneous fission source because a ${ }^{252} \mathrm{Cf}$-source ionization chamber is typically used in source-driven noise measurement. The basic fission chain process relating a count in a detector to the initiating source particle is depicted in Fig. A.1. The arrow represents an unknown number of fission events between the initial source event and the subsequent neutron detection.

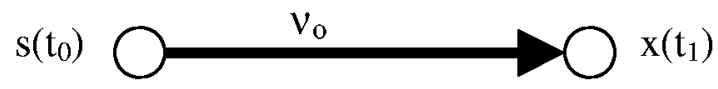

Fig. A.1. Fission chain for source event at time $t_{o}$ and detection event at time $t_{l}$.

The spontaneous fission of ${ }^{252} \mathrm{Cf}$ is a random process in that the spontaneous fission of an individual atom is independent of the spontaneous fission of any other atom. The spontaneous fission has an average rate defined as $F_{s}$. Therefore the probability of a spontaneous fission event $s$ at time $t_{0}$ in $d t_{0}$ that results in $n_{0}$ neutrons is simply

$$
p_{s}\left(t_{0}\right) d t_{0}=F_{s} p\left(n_{0}\right) d t_{0}
$$


where $p\left(n_{0}\right)$ is the neutron multiplicity distribution for the source. Note that this probability must be multiplied by the probability, $e_{s}$, of detecting the spontaneous fission event. The conditional probability of subsequent neutron detection at time $t_{1}$ in $d t_{1}$ following the injection of $n_{0}$ neutrons at time $t_{0}$ in $d t_{0}$ is given as the product of the fission production probability and the detection probability

$$
p\left(t_{1} \mid t_{0}, n_{0}\right) d t_{1}=\frac{e_{1} n_{0} e^{-a\left(t_{1}-t_{0}\right)} d t_{1}}{\bar{n} \Lambda} .
$$

The efficiency, $e_{1}$, denoted in Eq. A.7 is the probability of neutron detection per fission. The joint probability of a count at time $t_{l}$ in $d t_{l}$ following a source event at time $t_{0}$ in $d t_{0}$ is given by

$$
\begin{aligned}
p\left(t_{1}, t_{0}\right) d t_{1} d t_{0} & =e_{s_{n_{0}}} \sum_{0}\left(n_{0}\right) F_{s} \frac{e_{1} n_{0} e^{-a\left(t_{1}-t_{0}\right)}}{\bar{n} \Lambda} d t_{1} d t_{0} \\
& =e_{s} F_{s} \frac{e_{1} \bar{n}_{0} e^{-a\left(t_{1}-t_{0}\right)}}{\bar{n} \Lambda} d t_{1} d t_{0} .
\end{aligned}
$$

The joint probability depends only on the time difference between the source event an the detection event $\left(t=t_{I}-t_{0}\right)$ and can be simplified as

$$
p(t) d t=\frac{e_{s} e_{1} \bar{n}_{0} F_{s} e^{-a(t)}}{\bar{n} \Lambda} d t .
$$

The source-detector covariance function that is the same as the randomly pulsed neutron measurement is thus defined as

$$
C_{s x}(t)=\frac{e_{s} e_{1} \bar{n}_{0} F_{s} e^{-a(t)}}{\bar{n} \Lambda}
$$

The second part of the source-driven noise measurement is the Rossi- $\alpha$ measurement that involves the time correlation of detection events between detectors. The derivation that follows is similar to that provided by Mattingly ${ }^{11}$ with explicit description of the fission chain process. There are two means by which a spontaneous fission source may contribute to the correlation between two detectors. First two neutrons from a spontaneous fission event may create fission chains that are related in time, and secondly a single source neutron may induce a fission event that leads to two fission chains that are related in time. These processes are depicted in Fig. A.2. Again the arrows represent an unknown number of fission events from the initiating event to the detection events. 

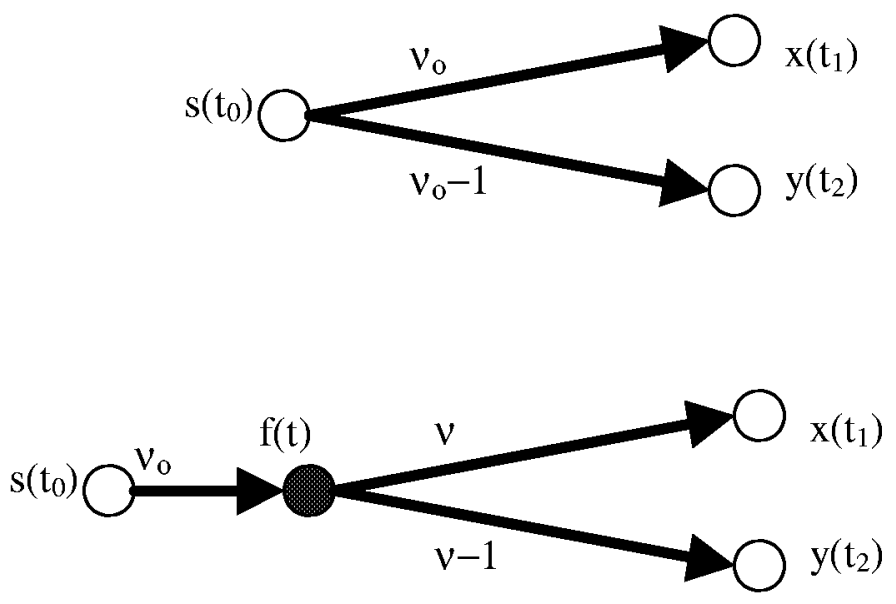

Fig. A.2. Fission chains for related detector counts.

The first process depicted in Fig. A.2 is the direct observation of fission chains caused by two neutrons from the spontaneous fission source. Again, the probability of a source spontaneous fission at time $t_{0}$ in $d t_{0}$ resulting in the emission of $n_{0}$ neutrons is given by Eq. A.6. The conditional probability of subsequent neutron detection at time $t_{I}$ in $d t_{I}$ following the injection of $n_{0}$ neutrons at time $t_{0}$ in $d t_{0}$ is given as the product of the fission production probability and the detection probability

$$
p\left(t_{1} \mid t_{0}, n_{0}\right) d t_{1}=\frac{e_{1} n_{0} e^{-a\left(t_{1}-t_{0}\right)} d t_{1}}{\bar{n} \Lambda} .
$$

The probability of neutron detection at time $t_{2}$ in $d t_{2}$ following neutron detection at time $t_{1}$ in $d t_{l}$ and a source event at time $t_{0}$ in $d t_{0}$ that produces $n_{0}$ source neutrons is given by

$$
p\left(t_{2} \mid t_{1}, t_{0}, n_{0}\right) d t_{2}=\frac{e_{2}\left(n_{0}-1\right) e^{-a\left(t_{2}-t_{0}\right)} d t_{2}}{\bar{n} \Lambda}
$$

The term $n_{0^{-}} 1$ appears in Eq. A.12 because one source neutron was required to produce the first fission chain. The joint probability of neutron detection events at time $t_{2}$ in $d t_{2}$ and at time $t_{I}$ in $d t_{I}$ following a source event at time $t_{0}$ in $d t_{0}$ resulting in the emission of $n_{0}$ neutrons is given as the product of Eqs. A.6, A.11, and A.12. The resulting expression is defined as follows 
$p\left(t_{2}, t_{1}, t_{0}, n_{0}\right) d t_{2} d t_{1} d t_{0}=\frac{e_{1} e_{2} F_{s} p\left(v_{0}\right) v_{0}\left(n_{0}-1\right) e^{-a\left(t_{2}+t_{1}-2 t_{0}\right)} d t_{2} d t_{1} d t_{0}}{(\bar{n} \Lambda)^{2}}$.

The probability of a chain-related count at times $t_{2}$ in $d t_{2}$ and $t_{1}$ in $d t_{1}$ is the integral of Eq. A.13 over all time for the initial source fission event up to the first detection event at time $t_{l}$. The probability is also summed over all possible number of neutrons from the source. The resulting expression is

$$
\begin{aligned}
p\left(t_{2}, t_{1}\right) d t_{2} d t_{1} & =e_{1} e_{2} F_{s_{n_{0}}} p\left(v_{0}\right) v_{0}\left(n_{0}-1\right) \int_{-\infty}^{t_{1}} \frac{e^{-a\left(t_{2}+t_{1}-2 t_{0}\right)} d t_{0} d t_{1} d t_{2}}{(\bar{n} \Lambda)^{2}} . \\
& =e_{1} e_{2} \overline{v_{0}\left(n_{0}-1\right)} F_{s} \frac{e^{-a\left(t_{2}-t_{1}\right)}}{2 a(\bar{n} \Lambda)^{2}} d t_{1} d t_{2}
\end{aligned}
$$

The second process by which a source neutron can contribute to correlation events at two different times is also depicted in Fig. A.2. In this process, a source neutron produced at time $t_{0}$ in $d t_{0}$ induces a fission event at time $t$ in $d t$. The conditional probability of a fission event at time $t$ in $d t$ following the emission of $n_{0}$ source neutrons at time $t_{0}$ in $d t_{0}$ is given by

$$
p_{f}\left(t \mid t_{0}, n_{0}\right) d t=p(n) \frac{n_{0} e^{-a\left(t-t_{0}\right)} d t}{\bar{n} \Lambda} .
$$

The probability of a fission event at time $t$ in $d t$ that results in the probability of $p(n)$ neutrons being emitted is the integral of the product of Eqs. A.6 and A.15

$$
\begin{aligned}
p_{f}(t) d t & =p(n) F_{s_{n_{0}}} p\left(n_{0} n_{0} \int_{-\infty}^{t} \frac{e^{-a\left(t-t_{0}\right)} d t d t}{\bar{n} \Lambda}\right. \\
& =p(n) \frac{F_{s} \overline{n_{0}}}{\bar{n} \Lambda a} .
\end{aligned}
$$


The conditional probability of subsequent neutron detection at time $t_{l}$ in $d t_{l}$ following the production of $n$ neutrons from fission at time $t$ in $d t$ is given as the product of the fission production probability and the detection probability

$$
p\left(t_{1} \mid t, n\right) d t_{1}=\frac{e_{1} n e^{-a\left(t_{1}-t\right)} d t_{1}}{\bar{n} \Lambda} .
$$

The probability of neutron detection at time $t_{2}$ in $d t_{2}$ following neutron detection at time $t_{1}$ in $d t_{l}$ and a fission event at time $t$ in $d t$ is given by

$$
p\left(t_{2} \mid t_{1}, t, n\right) d t_{2}=\frac{e_{2}(n-1) e^{-a\left(t_{2}-t\right)} d t_{2}}{\bar{n} \Lambda} .
$$

Again, the term $n-1$ appears in Eq. A.18 because one fission neutron was required to produce the first fission chain. The joint probability of neutron detection events at time $t_{2}$ in $d t_{2}$ and at time $t_{1}$ in $d t_{1}$ following a fission event at time $t$ in $d t$ resulting in the emission of $n$ neutrons is given as the product of Eqs. A.16, A.17, and A.18. The resulting expression is defined as follows

$$
p\left(t_{2}, t_{1}, t, n\right) d t_{2} d t_{1} d t=\frac{e_{1} e_{2} F_{s} \bar{n}_{0} p(v) v(n-1) e^{-a\left(t_{2}+t_{1}-2 t\right)} d t_{2} d t_{1} d t}{a \Lambda(\bar{n} \Lambda)^{2}}
$$

The probability of a chain-related count at times $t_{2}$ in $d t_{2}$ and $t_{1}$ in $d t_{1}$ is the integral of Eq. A.19 over all time for the fission event up to the first detection event at time $t_{l}$. The probability is also summed over all possible number of neutrons from the fission event. The resulting expression is

$$
\begin{aligned}
p\left(t_{2}, t_{1}\right) d t_{2} d t_{1} & =e_{1} e_{2} \frac{n_{0} F}{a \Lambda} \sum_{n_{0}} p(v) v(n-1) \int_{-\infty}^{t_{1}} \frac{e^{-a\left(t_{2}+t_{1}-2 t\right)} d t d t_{1} d t_{2}}{(\bar{n} \Lambda)^{2}} . \\
& =e_{1} e_{2} \frac{\bar{n}_{0} F_{s}}{a \Lambda} \frac{e^{-a\left(t_{2}-t_{1}\right)}}{2 a(\bar{n} \Lambda)^{2}} d t_{1} d t_{2}
\end{aligned}
$$

The total probability of a chain-related count at times $t_{2}$ in $d t_{2}$ and $t_{1}$ in $d t_{1}$ is the sum of Eqs. A.14 and A.20. The resulting expression is simplified to 


$$
p\left(t_{2}, t_{1}\right) d t_{2} d t_{1}=e_{1} e_{2}\left[\overline{v(n-1)} \frac{\bar{n}_{0} F_{s}}{a \Lambda}+\overline{v_{0}\left(n_{0}-1\right)} F_{s}\right] \frac{e^{-a\left(t_{2}-t_{1}\right)}}{2 a(\bar{n} \Lambda)^{2}} d t_{1} d t_{2} .
$$

This probability depends only on the time difference between the detection events and can be simplified to

$$
p(t) d t=e_{1} e_{2}\left[\overline{v(n-1)} \frac{\bar{n}_{0} F_{s}}{a \Lambda}+\overline{v_{0}\left(n_{0}-1\right)} F_{s}\right] \frac{e^{-a|t|}}{2 a(\bar{n} \Lambda)^{2}} d t .
$$

Note that in Eq. A.22 that the absolute values of the time difference is specified because the detection events can occur in any order. If a system contains other neutron sources besides the ${ }^{252} \mathrm{Cf}$ source, these sources will also contribute to the correlation between detectors. For example, plutonium systems contain a significant inherent spontaneous fission source. This inherent spontaneous fission source would have similar contributions as those defined for the ${ }^{252} \mathrm{Cf}$ source. The probability of chain-related counts at times $t_{2}$ in $d t_{2}$ and $t_{1}$ in $d t_{1}$ due to the inherent spontaneous fission source is

$$
p(t) d t=e_{1} e_{2}\left[\overline{v(n-1)} \frac{\overline{n_{I}} F_{I}}{a \Lambda}+\overline{v_{I}\left(n_{I}-1\right)} F_{I}\right] \frac{e^{-a|t|}}{2 a(\bar{n} \Lambda)^{2}} d t .
$$

The term $n_{I}$ is the number of inherent spontaneous fission neutrons, and $F_{I}$ is the inherent spontaneous fission source rate. The detector-detector covariance function including both the ${ }^{252} \mathrm{Cf}$ source and an inherent spontaneous fission source would be defined as

$$
\begin{aligned}
C_{x y}(t) & =e_{1} e_{2}\left[\overline{v(n-1)} \frac{\overline{n_{0}} F_{s}}{a \Lambda}+\overline{v_{0}\left(n_{0}-1\right)} F_{S}+\overline{v(n-1)} \frac{\overline{n_{I}} F_{I}}{a \Lambda}+\overline{v_{I}\left(n_{I}-1\right)} F_{I}\right] \\
& \bullet \frac{e^{-a|t|}}{2 a(\bar{n} \Lambda)^{2}} .
\end{aligned}
$$

The detector-detector covariance function defined by Eq. A.24 is the same as the Rossi- $\alpha$ measurement. This measurement would be referred to as a two-detector Rossi- $\alpha$ measurement. If the measurement is performed with a single detector, the measurement is referred to as a single-detector Rossi- $\alpha$ measurement and an additional term appears in the expression for the detector auto covariance function. The single-detector auto covariance is 


$$
\begin{aligned}
C_{x x}(t) & =e_{1} e_{1}\left[\overline{v(n-1)} \frac{\overline{n_{0}} F_{s}}{a \Lambda}+\overline{v_{0}\left(n_{0}-1\right)} F_{s}+\overline{v(n-1)} \frac{\overline{n_{I}} F_{I}}{a \Lambda}+\overline{v_{I}\left(n_{I}-1\right)} F_{I}\right] \\
& \bullet \frac{e^{-a|t|}}{2 a(\bar{n} \Lambda)^{2}}+e_{1} d(t)\left[\frac{\overline{n_{0}} F_{S}}{a \Lambda}+\frac{\overline{n_{I}} F_{I}}{a \Lambda}\right] .
\end{aligned}
$$

The second term appears in Eq. A.25 because the detector signal is always correlated with itself. The source-driven noise measurement is based on both the randomly pulsed neutron and Rossi- $\alpha$ measurements. The simplistic point models for these measurements have been derived to demonstrate the dependencies of these measurements on the moments of prompt neutrons from fission, the prompt neutron decay constant, and the neutron generation time. The accidental coincidences between the source and detectors and between detectors have been ignored in the previous derivations to simplify the description of the physics of the measurements. Obviously the accidental coincidence rate will be a product of the count rates of the different detectors times the time interval over which the counts are acquired. 

S. J. Ball
L. G. Chiang
R. G. Gilliland
K. H. Guber
C. M. Hopper
L. C. Leal
J. K. Mattingly
D. W. McDonald
J. T. Mihalczo
R. B. Oberer
C. V. Parks
R. B. Perez
R. W. Roussin
A. W. Trivelpiece
T. E. Valentine (10)
R. M. Westfall
J. D. White
M. C. Wright

\section{EXTERNAL DISTRIBUTION}

\section{Department of Energy}

R. L. Dintaman

Office of RD\&T Facilities, DP-13

United States Department of Energy

19901 Germantown Road

Germantown, MD 20874

D. Y. Chung

System Engineering Division, DP-45

United States Department of Energy

19901 Germantown Road

Germantown, MD 20874 


\section{EXTERNAL DISTRIBUTION (CONT'D.)}

\section{Idaho National Engineering and Environmental Laboratory}

J. B. Briggs

Idaho National Engineering \& Environmental Laboratory

2525 N. Fremont

P. O. Box 1625

Idaho Falls, ID 83415-3855

Los Alamos National Laboratory

R. E. Anderson

Los Alamos National Laboratory,

NIS-6, MS J562, TA-18

Los Alamos, NM 87545

C. A. Goulding

Los Alamos National Laboratory, NIS-6, MS J562, TA-18

Los Alamos, NM 87545

R. G. Sanchez

Los Alamos National Laboratory, NIS-6, MS J562, TA-18

Los Alamos, NM 87545

T. P. McLaughlin

Los Alamos National Laboratory

ESH-6, MS F691

Los Alamos, NM 87545

\section{France}

B. Verrey

Institute for Nuclear Safety and Protection

Valduc SRCS

21120 IS-SUR-TILLE, France 


\section{EXTERNAL DISTRIBUTION (CONT'D.)}

\section{Japan}

Y. Mihyoshi

Criticality Safety Laboratory

Department of Fuel Cycle Safety Research

Japan Atomic Energy Research Institute

Tokai-mura, Naka-gun, Ibaraki-ken

319-11 Japan

T. Yamamoto

Criticality Safety Laboratory

Department of Fuel Cycle Safety Research

Japan Atomic Energy Research Institute

Tokai-mura, Naka-gun, Ibaraki-ken

319-11 Japan

Ichiro Nojiri

Health and Safety Division

PNC Tokai Works

4-33, Muramatsu, Tokai-mura

Ibaraki-ken, Japan, 319-11 